\title{
Towards the Interpretability of Deep Learning Models for Human Neuroimaging
}

Simon M. Hofmann *1,2,3, Frauke Beyer ${ }^{1,3}$, Sebastian Lapuschkin ${ }^{2}$, Markus Loeffler ${ }^{4}$, KlausRobert Müller ${ }^{5,6,7,8,9}$, Arno Villringer ${ }^{1,3,10,11}$, Wojciech Samek ${ }^{2,9}$, A. Veronica Witte $^{*, 1,3}$

${ }^{1}$ Department of Neurology, Max Planck Institute for Human Cognitive and Brain Sciences, 04103 Leipzig, Germany

${ }^{2}$ Department of Artificial Intelligence, Fraunhofer Institute Heinrich Hertz, 10587 Berlin, Germany

${ }^{3}$ Clinic for Cognitive Neurology, University of Leipzig Medical Center, 04103 Leipzig, Germany

${ }^{4}$ IMISE, University of Leipzig, 04103 Leipzig, Germany

${ }^{5}$ Machine Learning Group, Technical University Berlin, 10623 Berlin, Germany

${ }^{6}$ Department of Artificial Intelligence, Korea University, 02841 Seoul, South Korea

${ }^{7}$ Brain Team, Google Research, 10117 Berlin, Germany

${ }^{8}$ Max Planck Institute for Informatics, 66123 Saarbrücken, Germany

${ }^{9}$ BIFOLD - Berlin Institute for the Foundations of Learning and Data, 10587 Berlin, Germany

${ }^{10}$ MindBrainBody Institute, Berlin School of Mind and Brain, Humboldt-Universität zu Berlin, 10099 Berlin, Germany

${ }^{11}$ Center for Stroke Research, Charité - Universitätsmedizin Berlin, 10117 Berlin, Germany

${ }^{*}$ corresponding authors

Keywords: brain-age, structural MRI, explainable A.I., aging, FLAIR, SWI, diabetes 


\section{Abstract}

Brain-age (BA) estimates based on deep learning are increasingly used as neuroimaging biomarker for brain health; however, the underlying neural features have remained unclear. We combined ensembles of convolutional neural networks with Layer-wise Relevance Propagation (LRP) to detect which brain features contribute to BA. Trained on magnetic resonance imaging (MRI) data of a population-based study ( $n=2637,18-82$ years), our models estimated age accurately based on single and multiple modalities, regionally restricted and whole-brain images (mean absolute errors 3.37-3.86 years). We find that BA estimates capture aging at both small and large-scale changes, revealing gross enlargements of ventricles and subarachnoid spaces, as well as lesions, iron accumulations and atrophies that appear throughout the brain. Divergence from expected aging reflected cardiovascular risk factors and accelerated aging was more pronounced in the frontal lobe. Applying LRP, our study demonstrates how superior deep learning models detect brain-aging in healthy and atrisk individuals throughout adulthood. 


\section{Introduction}

With the advent of large-scale magnetic resonance imaging (MRI) studies (e.g., ukbiobank, Sudlow et al., 2015; LIFE, Loeffler et al., 2015), the estimation of brain age (BA), and its contrast to the chronological age of a person (diverging BA, DBA), have become an increasingly predictive imaging marker for brain health. Higher DBA relates to accelerated cognitive decline, pathologies such as Alzheimer Disease (AD), hypertension and type 2 diabetes, as well as other lifestyle-related cardiovascular risk factors (Dadi et al., 2020; Franke \& Gaser, 2019). However, underlying alterations of neural structures reflecting the relationship between BA and such factors are not well known. BA has been linearly estimated on predefined neuroimaging outcomes (e.g., cortical thickness maps Liem et al., 2017). Yet, feature extraction and preprocessing could lead to overconfidence w.r.t., or to the dismissal of, neural properties that can be relevant to BA. In contrast, deep learning (DL) models, specifically convolutional neural networks (CNNs; Ji et al., 2013; LeCun et al., 1989) are trained on raw data and provide more precise BA estimates (Cole et al., 2017; Cole \& Franke, 2017). Particularly on large MRI datasets CNNs converge to a minimal mean absolute error (MAE) of 2.14 years (Peng et al., 2021; see also Bashyam et al., 2020; Dinsdale et al., 2021; Feng et al., 2020; Jonsson et al., 2019; Kolbeinsson et al., 2020; Levakov et al., 2020). Despite these advantages, their complex architectures restrict straightforward interpretations of which image features drive their estimates, known as the black-box problem (Samek et al., 2019, 2021). Several methods have been proposed to open the black-box (Samek et al., 2021), such as perturbation and gradient techniques (Baehrens et al., 2010; Simonyan et al., 2014; Smilkov et al., 2017; Sundararajan et al., 2017; Zeiler \& Fergus, 2014; Zintgraf et al., 2017), which also have been applied for BA predictions (Levakov et al., 2020). While many of these methods highlight input areas or intermediate feature maps that are relevant for the prediction, they do not indicate whether this information increases or decreases the predictor output. For the continuous case of BA estimates this means that neither the pace of aging processes (i.e., DBA), nor the state of their progression (BA) can be inferred from computed saliency maps. 
Conversely, the Layer-wise Relevance Propagation algorithm (LRP) highlights relevant areas in the input (image) that both favor and dismiss corresponding output decisions (Bach et al., 2015; Lapuschkin et al., 2019; Montavon et al., 2018). LRP has been successfully used with DL in MRI-based classification tasks (Böhle et al., 2019; Eitel et al., 2019; A. W. Thomas et al., 2019). However, the biological alterations that underlie aging are continuous in nature, which raises more challenges for both the DL model, and, consequently, its interpretation.

Here, we therefore aimed to provide a novel, openly available analysis pipeline extrapolating from a proof-of-concept simulation study to the implementation of superior CNNs on multimodal MRI with the explanation algorithm LRP. Specifically, we asked which neurostructural features drive individual predictions and whether BA truly captures biological aging processes. On a group level we explored, how DBA is modulated by cardiovascular risk factors, and how this relationship manifests in distinct neural features. Based on previous findings, we hypothesized that BA relies on grey matter atrophy which include (pre)frontal and mesiotemporal cortex and cerebellum, and that risk factors such as obesity, hypertension and type 2 diabetes correlate with higher DBA, reflected in augmented vascular pathologies such as higher white matter lesion load. Importantly, opening the black box of DL image analysis is expected to reveal novel features of MRI-based neuronal properties that contribute to BA estimates, and thus advance our knowledge of brain health in aging.

\section{Results}

We implemented two types of multi-level ensembles (MLens, Fig. 1) on three clinically relevant MRI modalities (T1-weighted, fluid-attenuated inversion recovery, FLAIR, and Susceptibility Weighted Imaging, SWI) of a well-characterized population-based cohort study (LIFE-Adult; Loeffler et al., 2015; age range 18-82 years, $n=2016$ ).

Briefly, MLens type i was trained on whole brain MRI with a sub-ensemble for each sequence with ten 3D-CNN models (base models, BM). Sub-ensembles served to extract information on model certainty and to compute more robust BA estimates. To additionally explore the 
contribution of three distinct brain regions (cortical, sub-cortical structures, and cerebellum) to the BA estimate, MLens type ii was trained on $3^{2}$ combinations of the MRI sequences and the brain regions, while employing 5 BMs for each combination.

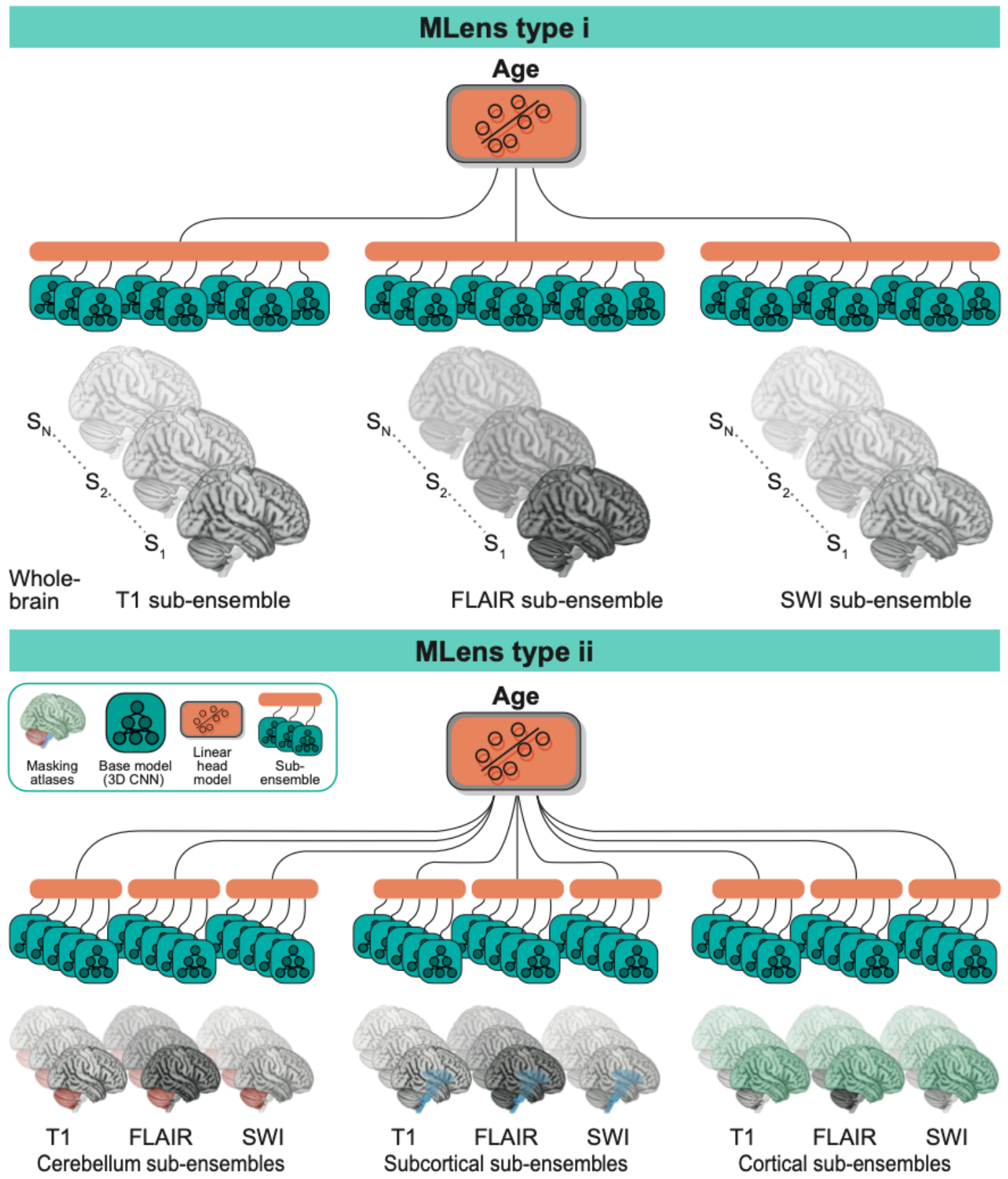

Fig. 1. Multi-level ensembles (MLens) MLens trained on the different MRI sequences (T1, FLAIR, SWI; top: MLens type i), and their combinations with 3 brain regions (bottom: MLens 
type ii). The predictions of the sub-ensembles of each MLens on the test set were used to train and evaluate the top-level linear head model.

\section{Model prediction performances}

Model performances (in mean absolute error, MAE)

\begin{tabular}{|c|c|c|c|c|c|}
\hline \multirow[t]{2}{*}{ Ensembles } & \multirow[t]{2}{*}{ Head model } & \multicolumn{4}{|c|}{ Base models } \\
\hline & & mean $_{M A E} \pm S D$ & $\min _{M A E}$ & $\max _{M A E}$ & $N_{\text {bm, MLens }}$ \\
\hline Multi-level ensemble (type i) & 3.86 & - & - & - & 30 \\
\hline T1 sub-ensemble & 4.11 & $4.66 \pm 0.28$ & 4.03 & 5.50 & 10 \\
\hline FLAIR sub-ensemble & 4.16 & $4.64 \pm 0.24$ & 3.97 & 5.27 & 10 \\
\hline SWI sub-ensemble & 5.74 & $6.54 \pm 0.63$ & 4.93 & 7.88 & 10 \\
\hline Multi-level ensemble (type ii) & 3.37 & - & - & - & 45 \\
\hline Cortical-T1 sub-ensemble & 4.64 & $5.33 \pm 0.31$ & 4.67 & 6.09 & 5 \\
\hline Cortical-FLAIR sub-ensemble & 4.27 & $4.91 \pm 0.35$ & 4.05 & 5.81 & 5 \\
\hline Cortical-SWI sub-ensemble & 5.87 & $6.70 \pm 0.54$ & 5.52 & 7.88 & 5 \\
\hline Sub-Cortical-T1 sub-ensemble & 4.53 & $5.46 \pm 0.46$ & 4.28 & 6.65 & 5 \\
\hline Sub-Cortical-FLAIR sub-ensemble & 3.89 & $4.66 \pm 0.44$ & 3.94 & 5.97 & 5 \\
\hline Sub-Cortical-SWI sub-ensemble & 4.79 & $5.73 \pm 0.52$ & 4.64 & 7.02 & 5 \\
\hline Cerebellum-T1 sub-ensemble & 5.27 & $6.17 \pm 0.40$ & 5.50 & 7.40 & 5 \\
\hline Cerebellum-FLAIR sub-ensemble & 4.83 & $5.49 \pm 0.40$ & 4.76 & 6.62 & 5 \\
\hline Cerebellum-SWI sub-ensemble & 7.21 & $8.22 \pm 0.74$ & 6.75 & 10.55 & 5 \\
\hline
\end{tabular}

Tab. 1. Prediction performances of both types of multi-level ensembles (MLens type $i$,

ii) and their respective sub-ensembles and 3D-CNN base models $(\mathrm{bm})$, measured in mean absolute error (MAE). To receive an age estimate for each subject, MLens were trained in a 10-fold cross-validation approach such that each subjects lies once in an unseen test set.

The MLens type i had a MAE of 3.86 and performed slightly better than all its sub-ensembles $\left(\mathrm{MAE}_{\mathrm{T} 1}=4.11, \mathrm{MAE}_{\mathrm{FLAIR}}=4.16, \mathrm{MAE}_{\mathrm{SWI}}=5.74 ;\right.$ Tab. 1). The MLens type ii had a smaller MAE of 3.37 (see Fig. 2 for prediction accuracy and model uncertainty) and was again superior to the performances of its sub-ensembles (Tab. 1). Between both MLens, there were highly significant correlations between their predictions $(R=0.97, p<0.001)$ and their prediction errors $(R=0.73, p<0.001)$ on the test set. Note that these models were trained with leaky 
rectified linear units (ReLUs), while models trained with standard ReLUs performed worse (MLens type i, MAE = 3.88; MLens type ii, MAE = 3.69; see T1 in the supplementary material).
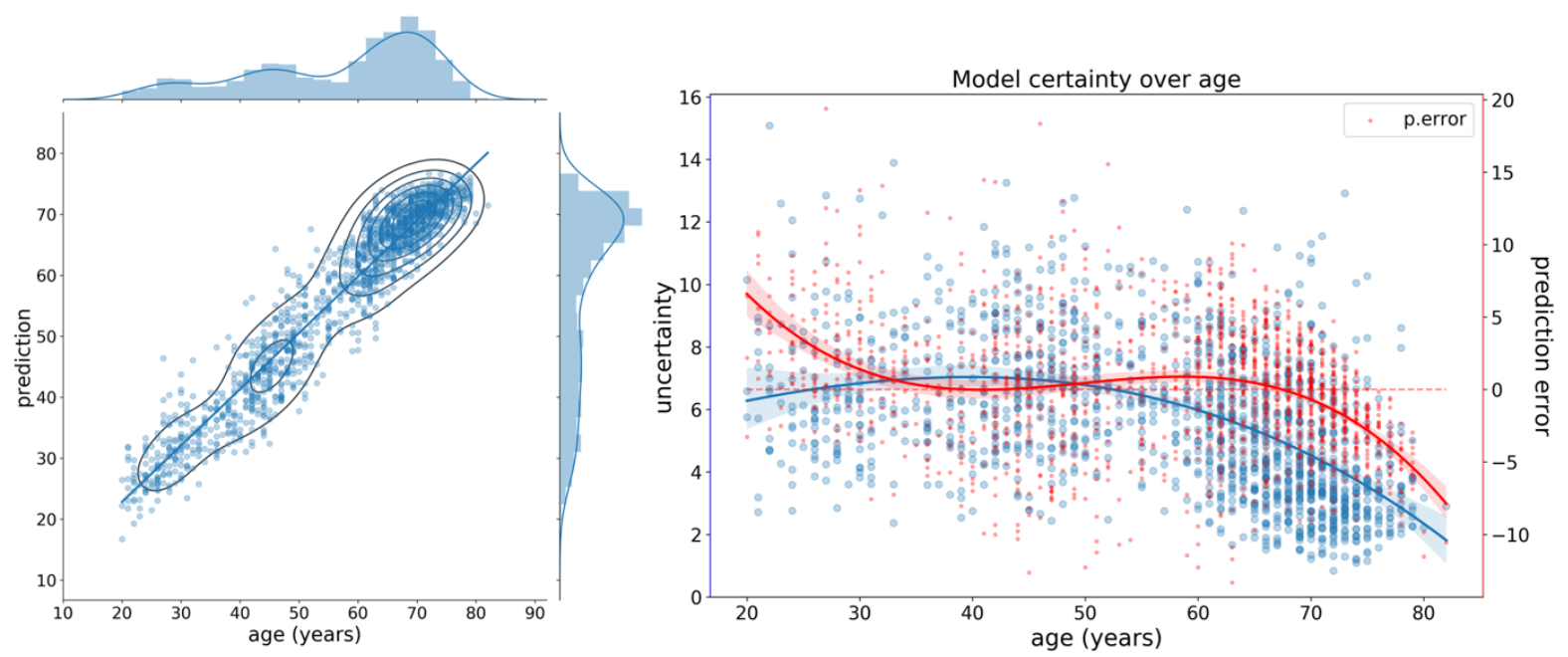

Fig. 2. Brain-age prediction performance and model certainty of deep learning-based multi-level ensembles (MLens) combining clinically relevant MRI sequences Left panel: test set predictions of the MLens type ii), trained on $3^{2}$ combinations of MRI sequences (T1, FLAIR, SWI) and brain regions (cortical, subcortical structures, and cerebellum). Right: prediction error (red) and model uncertainty (blue) per participant. Model uncertainty is measured as the width of the 95\%-confidence interval across the predictions of the subensembles. The modulation of both variables as function of age was modeled with a $3^{\text {rd }}$ order polynomial (red and blue lines). Both plots are produced over the concatenated test sets of the 5-fold-cross-validation, which were used to evaluate the top-level head models of the ensemble.

\section{Relevance maps of model predictions}

To verify the behavior of the LRP algorithm and its correct interpretation in a regression task, we first performed a simulation study.

The CNN model for the simulation task corresponded to a 2D-version of one base model in a MLens. It was trained on a simulation dataset of abstracted head models (tori; Fig. 3), in which 
aging was simulated as the accumulation of atrophies and lesions. The model had a MAE of 2.80 on the hold-out test set. The prediction model captured the simulated aging process in both its facets well, which is revealed by the LRP relevance maps (i.e., heatmaps) highlighting the inner and outer borders (atrophies), and the added lesions within the older tori (30+ years; Fig. 3). Areas, where atrophies can occur were considered as information bearing, i.e., they received both positive and negative relevance. Moreover, the model seemed to cluster information w.r.t. its regression task, which is represented in the unique sign of relevance over larger areas (see both tori on the right, Fig. 3). That is, while there were accumulations of atrophies at the border of some tori, the CNN also took adjacent lesions into account to aggregate the overall information in a specific region. Note that in some occasions this could lead to inversely weighted relevance in single pixels or small areas (see upper left part of green box in Fig. 3). The sum over all distributed relevance $r$ is a proxy for the final model prediction $\left(p=b_{t}+\sum r\right)$. If it is positive, the prediction $p$ is greater than the initiated model target bias $\left(b_{t}\right.$; here, set to the mean age of the sample: $b_{t}=51.1$ years), and vice versa for the negative case. Hence, the summed relevance represented the evidence over the whole image that the model accumulated to make its prediction. 

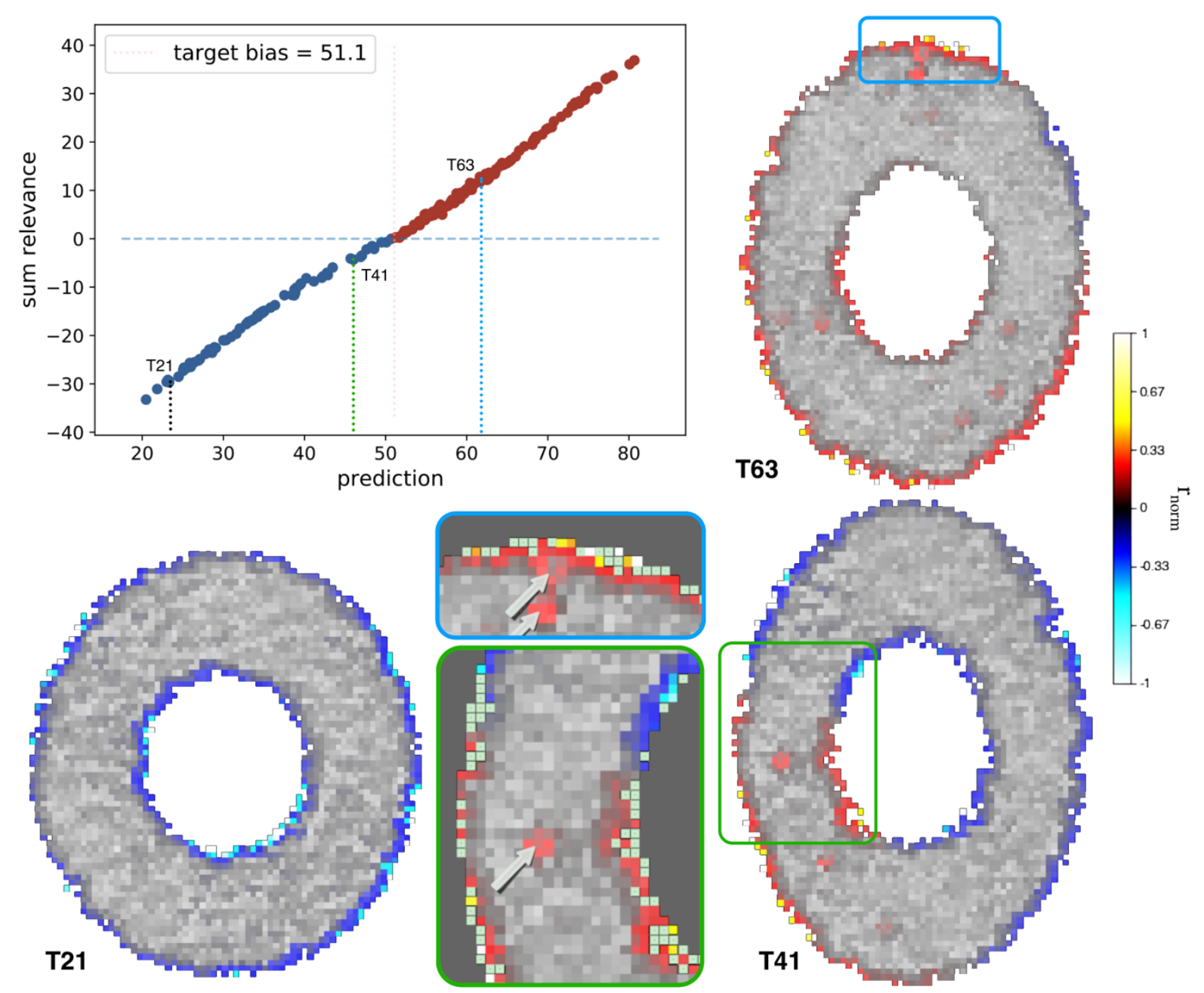

T41

Fig. 3. Analysis of simulated aging in artificial tori Top left: Summed relevance per predicted sample in the test dataset, reflecting the model prediction relative to the sample mean (i.e., target bias $=51.1$ years). Bottom left and right column: three image samples of tori (T[age]) with their corresponding LRP relevance maps overlaid. Gray boxes: Details of relevance maps of tori T41 (green) and T63 (blue), respectively. Here, arrows indicate added lesions, while mint-green pixels at the inner and outer borders of the tori indicate ground-truth atrophies. Note that intact matter is predominantly attributed with negative (blue-turquois) relevance, indicating a younger age, while lesioned or atrophied matter is attributed with positive (red-yellow) relevance pointing to an older age. Color coding: relevance values were symmetrically clipped around zero at the 0.99-percentile, then normalized $\left(\mathrm{r}_{\mathrm{norm}} \in[-1,1]\right)$ and the corresponding colormap was multiplied by a factor of 5 for better contrasts. Note, while 
the model predictions are continuous, we deliberately decided for a binary color scaling to better contrasts the lower (young) and upper (old) bound of the regression.

\section{Relevance maps of the aging brain in individuals}

Qualitative LRP analysis revealed individual relevance maps highlighting brain areas that voted for higher or lower BA predictions. Overall, we detected strong contributions from voxels in and around the ventricles and at the border from the brain to meningeal areas, independent of MRI sequence, while white-matter (WM) areas appeared to be less informative, except WM lesions in FLAIR images (Fig. 4a). In older participants, voxels covering cortical sulcal structures were often more relevant than in younger participants and voted more often in favor of older BA. Also, the corpus callosum, the brain stem and areas in and around the cerebellum appeared to be relevant structures, which the models gained information from for both younger and older participants.

Both types of MLens (whole-brain type i and region-based type ii) found similar brain structures important for their prediction (Fig. 4b). Visually most recognizable are areas around the ventricles, and subject specific sulci, e.g., in the cortex and cerebellum. 
bioRxiv preprint doi: https://doi.org/10.1101/2021.06.25.449906; this version posted August 26, 2021. The copyright holder for this preprint

(which was not certified by peer review) is the author/funder, who has granted bioRxiv a license to display the preprint in perpetuity. It is made available under aCC-BY-NC-ND 4.0 International license.
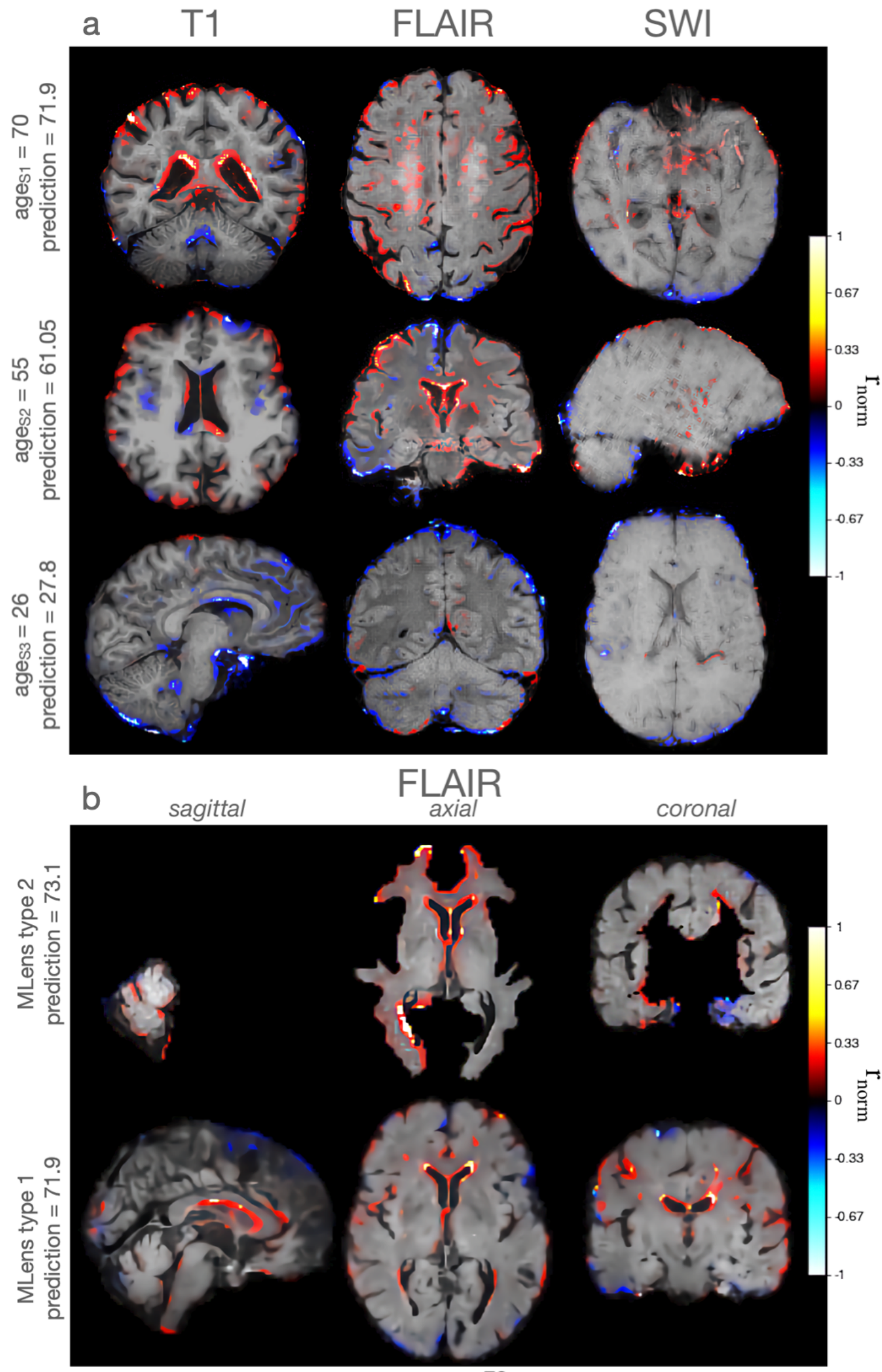

ages $1=70$ 
Fig. 4. Exemplary individual LRP heatmaps (a) of multi-level ensemble (MLens) type i. trained on whole brain data. Rows: three participants (S1-S3) drawn from different age groups. Columns: three MRI sequences (T1, FLAIR, SWI), individually sliced in all three axes. Next, to more global intact (mostly in young S3) or atrophied tissue (S1, S2), e.g., at the cortical surface, LRP also reveals smaller structures such as white-matter lesions (S1, FLAIR), vessel expansions and putative small iron depositions (S1, SWI) driving the BA estimation. Relevance maps per subject were aggregated over the base models of each sub-ensemble.

(b) LRP heatmaps of regional (top row, type ii) and whole-brain (bottom row, type i) MLens in elderly subject (S1). Here, models were trained on FLAIR data of cerebellum (left), subcortical structures (mid), and cortex (right), or of the whole-brain, respectively. For comparison, we warped the heatmap of whole-brain MLens type $i$ from subject space to MNI152 space (cf. top row in a). Color coding: as in Fig. 3.

\section{Statistical relevance maps over the adult lifespan}

Quantitively, permutation-based one-sample t-tests (5000 permutations, threshold-free cluster enhancement, TFCE, and family wise error, FWE-corrected $p \leq 0.05$ ) on combined relevance maps of the validation and test set $\left(n_{T 1}=n_{F L A I R}=402, n_{S W I}=314\right)$ of one MLens type i revealed that on average, in all $3 \mathrm{MRI}$ sequences, nearly the full brain contains meaningful information about BA (Fig. 5). The base models trained within the T1 sub-ensemble, gained most information in the lateral ventricle areas, corpus callosum, pre- and postcentral gyri in the motor and sensorimotor cortex, operculum, and all grey matter (GM) border areas including the frontal pole, temporal and visual poles and brainstem, and cerebellar borders. In the FLAIR sub-ensemble, most relevance was found around lateral ventricles, anterior temporal gyri, the pre- and postcentral gyri, and WM areas including cingulate gyrus, corpus callosum and fornix. Base models of the SWI sub-ensemble had a stronger focus on GM areas in the visual pole and occipital lobe, limbic areas, corpus callosum, WM fornix, internal capsule and on 
bioRxiv preprint doi: https://doi.org/10.1101/2021.06.25.449906; this version posted August 26, 2021. The copyright holder for this preprint

(which was not certified by peer review) is the author/funder, who has granted bioRxiv a license to display the preprint in perpetuity. It is made available under aCC-BY-NC-ND 4.0 International license.

subcortical nuclei and brainstem areas, including striatum, subthalamic nucleus, raphe and substantia nigra. 

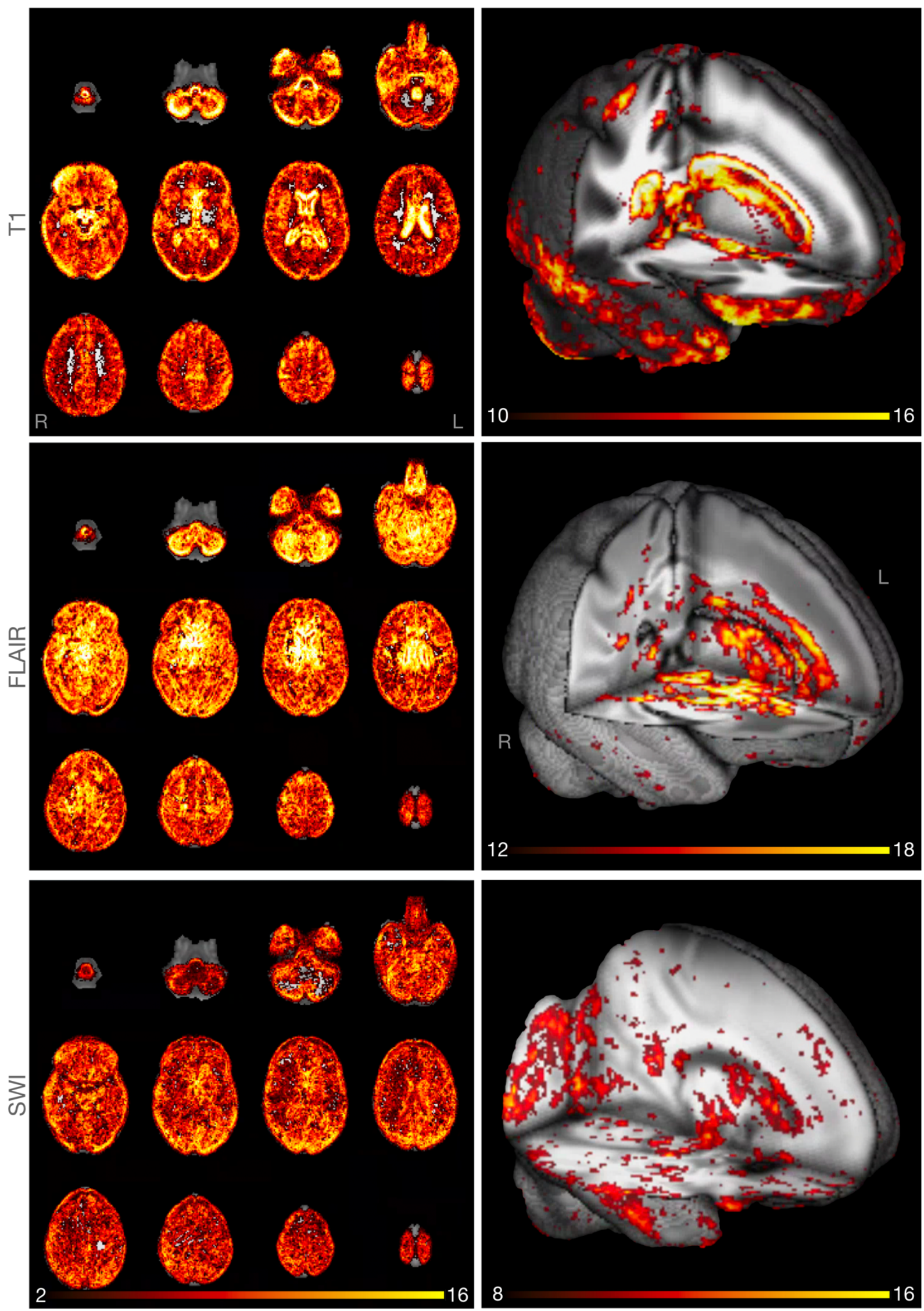

Fig. 5. Relevant areas for brain-age predictions across adult lifespan T-maps of one- 
sample t-test over aggregated, absolute relevance maps shown in several brain slices. Left column: $t(2,16) ;$ MNI152 z-axis range: 3-74. Right column: 3D-projection of t-maps focusing on higher t-values narrowly clipped for each MRI sequence, separately. Top row: tested on the T1 sub-ensemble (type $\left.i ; n=402, t_{\max }=23.61\right)$. Mid row: FLAIR sub-ensemble $(n=402$, $\left.t_{\max }=25.82\right)$. Bottom row: SWI sub-ensemble $\left(n=314, t_{\max }=16.07\right)$. The relevance were drawn from one of the MLens type i models.

Next, we compared the LRP heatmaps of the young (age $\leq 40$ years, $n=61$ ) versus older cohort (age $\geq 60$ years, $n=243$ ). Areas showing greater relevance in older compared to younger brains (TFCE, FWE-corrected $p \leq 0.5$ ) were found in the T1 sub-ensemble of MLens type i in lateral ventricles, corpus callosum, amygdala, cerebral WM, particularly paracingulate gyrus, opercular cortex, and (secondary) somatosensory cortex. For FLAIR, there were increased relevance values found in cerebellum (specifically, left and right crus I-II), caudate, inferior frontal gyrus, pars triangularis, insular cortex, and inferior parietal lobule. For the SWI sub-ensemble, frontal pole, frontal orbital cortex, Inferior frontal gyrus, pars triangularis, precuneus, basal nuclei including putamen and caudate, and occipital pole showed higher (i.e., positive) relevance on average (Fig. 6a). 
a
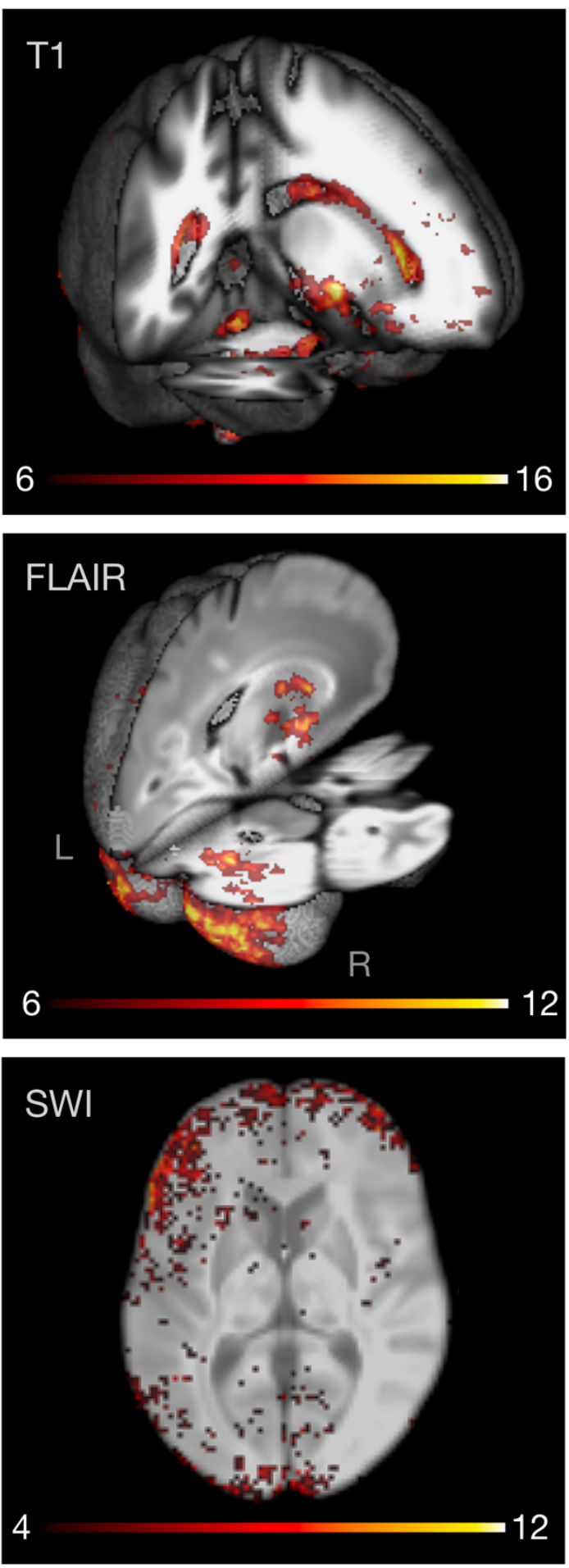

b
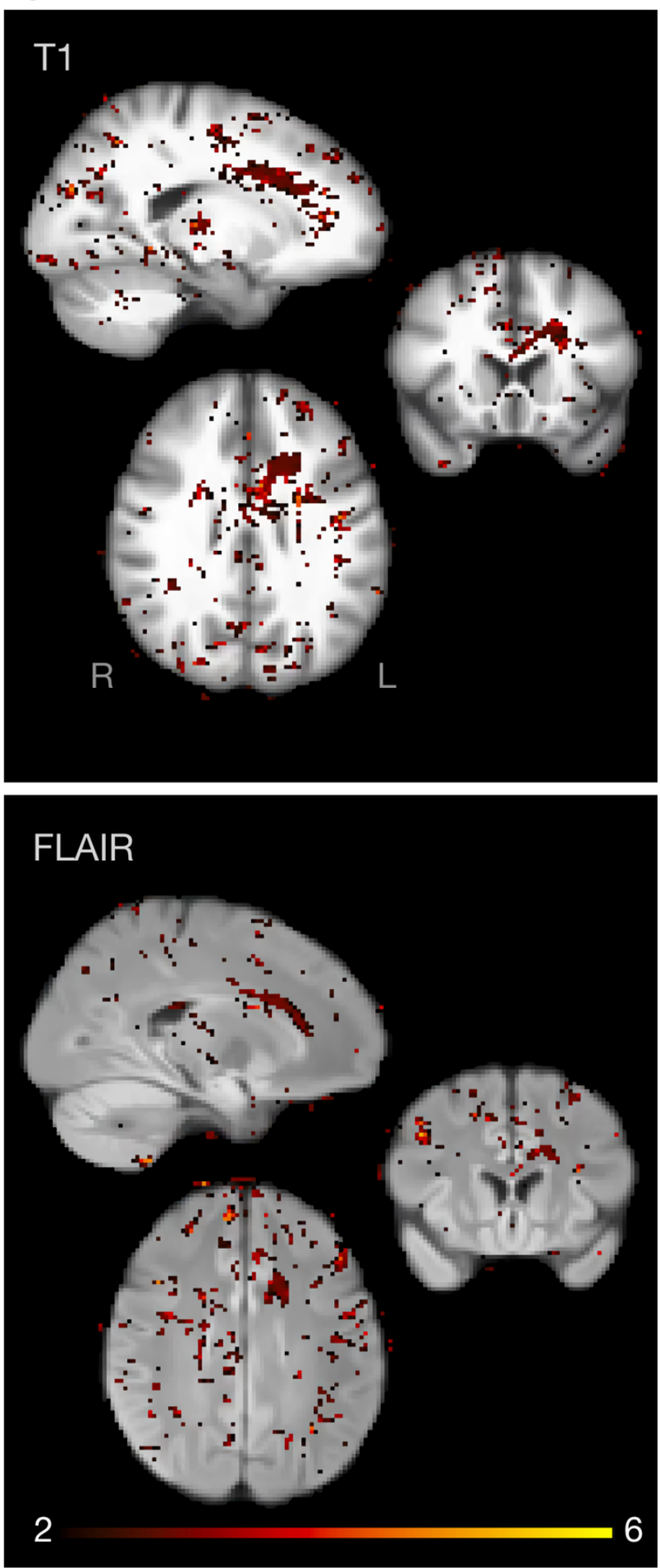

Fig. 6. a Contrastive relevance maps of young vs. elderly participants T-maps of twosample t-test over relevance maps in the young and elderly group. Here, testing shows in which areas the relevance is greater in elderly (age $\geq 60$ years) than in the young (age $\leq 40$ years) group. Relevance maps were aggregated within each sub-ensemble of one of the 
MLens type i models trained on T1 (top), FLAIR (mid), and SWI (bottom) data, respectively. $\boldsymbol{b}$

Contrastive relevance maps of healthy vs. diabetic participants T-maps $(2,6)$ of twosample t-test show in which areas the relevance is greater in participants with type 2 diabetes than in healthy controls of the older cohort (60-75 years). Relevance maps were aggregated within each sub-ensemble of one of the MLens type i models trained on T1 (top), and FLAIR (bottom). Note, only for T1 significant regional differences were found between the groups (see TFCE FWE-corrected maps in Fig. S2 in supplementary material). However, t-maps of T1 and FLAIR sub-ensembles show high correspondence (sliced in all three axes at $x=-18$., $y=18.1, z=28.1)$

\section{Relevance maps in diabetes and accelerated brain aging}

To explore the role of health-related risk factors on BA, we contrasted the LRP relevance maps of subjects with type 2 diabetes $(n=29)$ with healthy subjects $(n=217)$ in the age range of 50 to 75 years $\left(\right.$ mean $\left._{\text {age }}=65.61\right)$. For the T1 sub-ensemble (MLens, type $\mathrm{i}$ ), clusters of higher positive relevance (non-healthy > healthy) were found to be significant in the pre- and postcentral gyrus near the cortico-spinal tract in the primary motor cortex (TFCE, FWEcorrected $p \leq 0.011$ ), corpus callosum and cingulum (TFCE, FWE-corrected $p \leq 0.02$; see Fig. S2 in supplementary material). For the other two sub-ensembles (FLAIR, SWI), there were no clusters indicating significant regional differences. However, there was a high spatial correspondence between t-maps of the T1 and FLAIR sub-ensembles (Fig. 6b).

We further estimated the change in relevance maps as function of DBA, i.e., the signed prediction error, in an older cohort (age $\geq 50$, mean $_{\text {age }}=67.07, \mathrm{n}=134$ ), while controlling for age (as $2^{\text {nd }}$ order polynomial regression; cf. Fig. 2). Accordingly, all clusters indicating a significant association spatially corresponded to areas found in the BA analysis, however, accelerated aging (DBA) was more strongly related to higher relevance values in specific regions (see Fig. S3 in supplementary material): for the T1 sub-ensembles (MLens type i) this difference was found in frontal pole, brain stem, outer cerebellar boarders, WM including 
the cortical spinal tract, putamen, caudate, amygdala, pre- and post-central gyri, and cingulate gyri. For the FLAIR sub-ensemble, primarily posterior region showed significant associations, including occipital and parietal pole, lingual gyrus, and cerebellum (crus I and II, V, VI). Finally, for the SWI sub-ensembles, posterior and anterior regions showed significant associations, including the frontal pole, frontal orbital cortex, occipital pole, cerebellum (crus I and II, vermis VIII), but also some more left-lateral parieto-temporal WM structures close to putamen and operculum (for all sub-ensembles; TFCE, FWE-corrected $p \leq 0.05$ ).

\section{Diverging brain-age and its relationship to other biomarkers}

We found in the younger cohort (age $<45$ years) that higher DBA correlated with cardiovascular risk factors such as hypertension and hyperlipidemia according to exploratory correlation analyses, which were run on the hold-out test sets (Fig. 7). In older subjects (age $>60$ years) the most prevalent positive association of DBA was found with type 2 diabetes and accordingly, but weaker with glycated hemoglobin levels (HbA1c). BMI, waist-to-hip ratio and WM lesion load showed positive associations with DBA in participants almost across the full age range. Weak but relatively consistent trends appeared for the effect of gender (age > 30 years, where men had a higher BA on average) and the cognitive composite score, which showed a negative relationship with DBA. There was nearly no evident association between DBA and the presence or absence of an Apolipoprotein E epsilon 4 gene allele (APoE4), systolic blood pleasure or for higher education. Note, bivariate correlations were run in a sliding-age-window approach without adjusting for possible confounders; for multiple comparison a Bonferroni-correction was applied. 

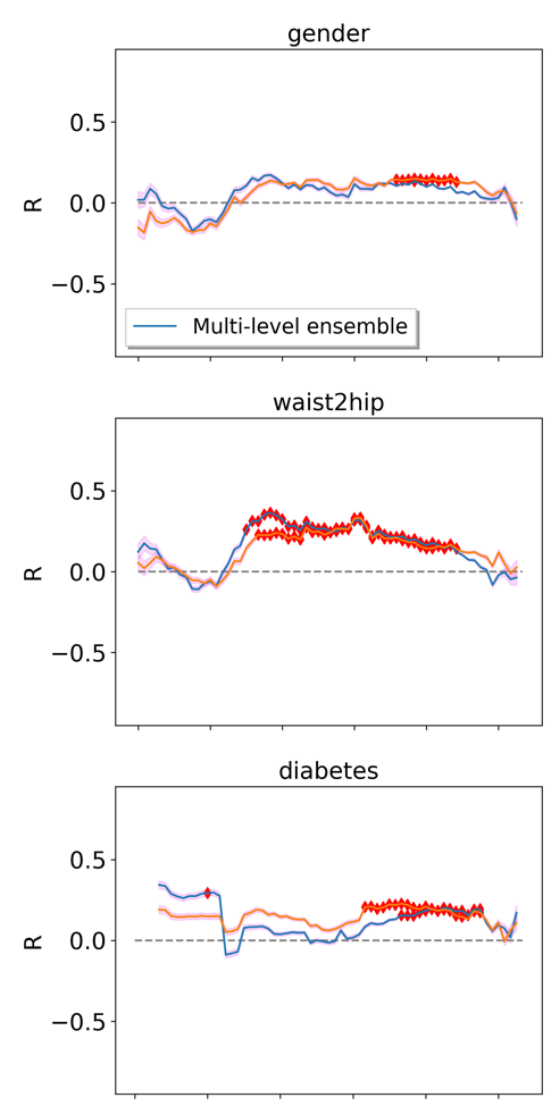

$\mathrm{HbAlc}$

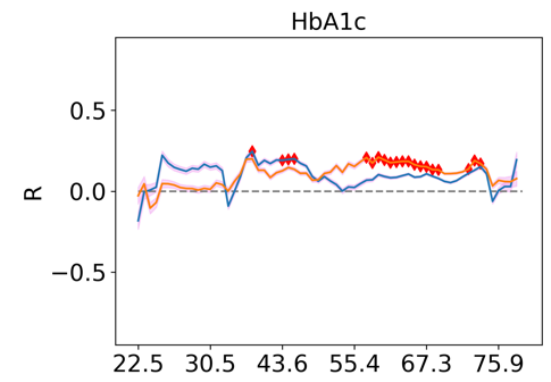

education (yrs)

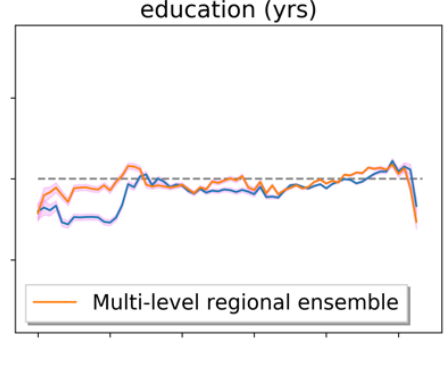

systolic BP

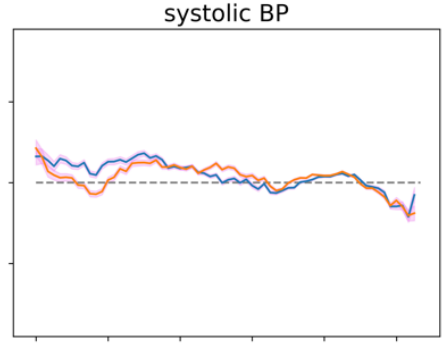

hyperlipidemia

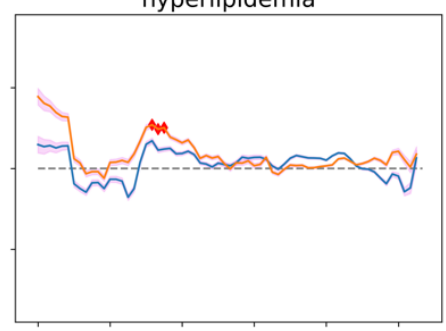

cognitive composite score

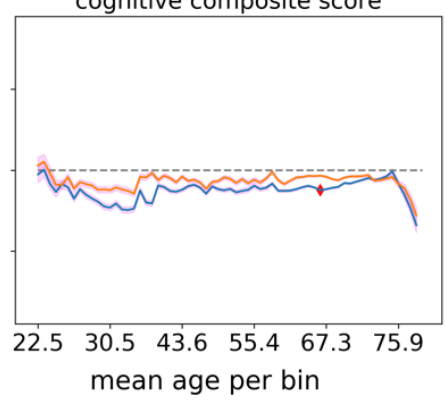

bmi

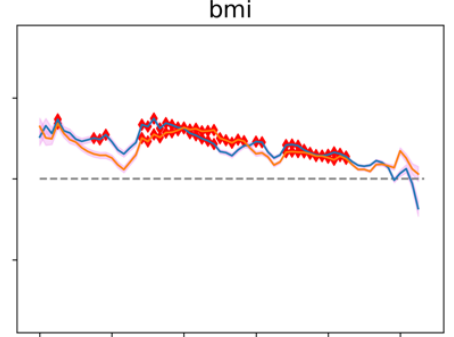

hypertension

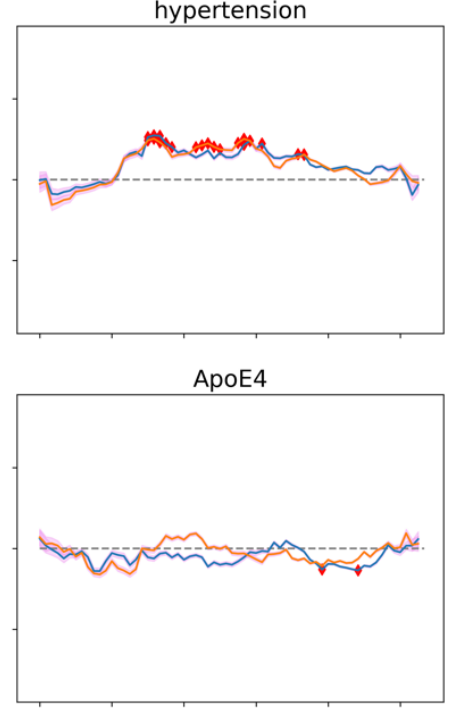

log lesionload-WM-ratio

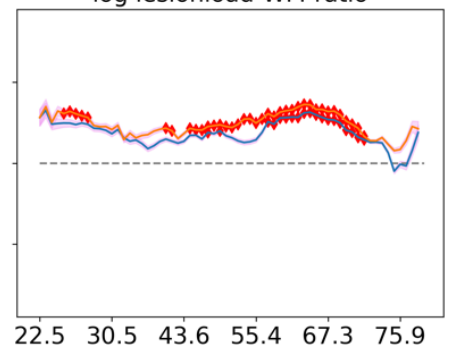

Fig. 7. Relationship of diverging brain-age to biomarkers and lifestyle factors Correlations $(R)$ in overlapping sliding windows (width 10 years) between prediction errors (DBA) of both models (blue: type i; orange: type ii MLens) and LIFE variables. Inverse width of the purple confidence band represents the number of participants per bin. Red rhombus: Bonferroni-corrected p-value $\leq 0.05$ per bin. Variables: education: time of education in years. bmi: body-mass-index. waist2hip: waist-to-hip-ratio. systolic BP: systolic blood pressure. APoE4: apolipoprotein epsilon 4 risk-allele carrier status. HbA1c: glycated hemoglobin. Log lesionload-WM-ratio: logarithmized ratio between number of lesions and white matter volume. 
Binary variables: hypertension, diabetes, hyperlipidemia, $A P o E 4:$ no $=0$, yes $=1$. gender: female $=0$, male $=1$.

\section{Discussion}

The estimation of age and deviance from expected aging trajectories from brain images is a difficult task that has been solved to a surprisingly high accuracy using various DL architectures (Bashyam et al., 2020; Cole et al., 2017; Cole \& Franke, 2017; Dinsdale et al., 2021; Feng et al., 2020; Jonsson et al., 2019; Kolbeinsson et al., 2020; Levakov et al., 2020; Peng et al., 2021). We provide a further dimension to this challenge, namely, the means to extract insight from the trained neural networks, such that neurobiological theories can be validated and novel hypotheses can be generated. Specifically, we demonstrate that our accurate estimates of continuous brain aging can be related back to neurostructural features, by employing interpretable (here using LRP), bias-free DL-ensemble models on multi-modal 3D-MRIs. Our analysis demonstrates that grey matter changes and atrophies detectable in the cortex, subcortex, cerebellum and brainstem, but also white matter lesions, and iron accumulations, as well as more global brain shrinkage represented in the larger size of ventricles and sulci drove the age estimates of the models. This happens to a degree that even parts of the brain and single MRI modalities (including SWI) led to accurate and comparable BA predictions. While voxels around the ventricular system and subarachnoid spaces were most informative for our model, the progression of aging and its pace (i.e., BA and DBA, respectively) could be related back nearly to the whole brain. Our simulation model furthermore revealed - as to be expected - that undamaged tissue (i.e., absence of atrophies and lesions) is associated with (young) age. From a methodological perspective, this demonstrated how the LRP algorithm can be integrated into a complex regression task on continuous aging, and how resulting relevance maps carry information about age-related changes. Moreover, we found that accelerated aging (DBA), which is associated with pathologies (here type 2 diabetes), shows relevant indicators in distinct brain areas, which 
could be differentiated by the complementary information from different MRI sequences and brain regions which we fed to the MLens models, leading to overall better prediction results. With this, we established a novel DL-based pipeline for MRI analysis, which leverages the predictive advantages of this model class while at the same time making its estimates interpretable for research and clinical applications.

\section{Opening the black-box of deep learning predictions}

To understand the estimates of our DL models, we applied the LRP algorithm, which provides directed, i.e., sign-specific, relevance maps in the input space. Since, at the point of model inference a classification problem is mathematically similar to a regression problem, LRP could be straight-forwardly adapted to the purpose of our study (see Methods). We successfully validated this approach in the regression domain according to a simulation study with a $2 \mathrm{D}$-version of the model architecture that we employed in the main study. We found that the DL model captures the simulated aging processes well by identifying the corresponding features. LRP comes with the advantage of being directional, i.e., it indicates not only that a certain input area is relevant for a given prediction, but also whether it provides information in direction to the upper (here old age) or lower bound (young age) of the regression problem (Fig. 3, 4). The sign of the sum of relevance (SoR) is arbitrary in this case, essential is the magnitude of the value. Here, we chose to set the bias at the output layer of the CNNs to the mean of the target variable (age). As a consequence, the SoR becomes negative for predictions lower, and positive for estimates higher than the bias. The model does not only capture the features that represent the aging process (atrophies and lesions), but also the absence of them. That is, for the young torus it attributes (here negative) relevance also to its intact surface and borders. Moreover, LRP shows that the CNN finds irregular occurring features (here lesions) which were randomly placed. However, the interpretation of the local attribution of relevance needs to be taken with caution, as we observed that the model often generalizes relevance over larger areas of the simulated tori. One possible 
explanation for this is that relevance might be clustered over bigger areas after being passed through the intermediate pooling and convolutional layers in the network, which aggregate information over increasingly larger areas in the computed feature maps. Then, later layers (usually fully connected layers) make decisions over these pooled regions by attributing relevance towards one of the main directions in the regression (Kohlbrenner et al., 2020).

\section{Normal and accelerated brain aging}

Applying LRP in the BA case shows that the DL models integrate information from the whole brain (Fig. 5). However, we see also that subject-specific structural properties are detected, specifically in the cortical surface areas, around ventricles, the corpus callosum, at the surface of the brain stem, and cerebellum, and distinct smaller regions in WM areas of the cortex. Ventricles are known to increase in size with age due to regional or global brain shrinkage (Earnest et al., 1979). Also, cortical surface (Jin et al., 2018; Kochunov et al., 2005; LeMay, 1984), the corpus callosum (Doraiswamy et al., 1991), cerebellum and basal ganglia (Raz et al., 2005, 2010) among others are subject to alterations. While Raz et al. $(2005,2010)$ found no age-related volume changes in, e.g., primary visual cortices and putamen, our model showed that both areas were relevant for the BA estimation across the full life-span, and ageindependent rate-of-aging (DBA) in the older cohort (age $\geq 50$ years). This may have several reasons: in contrast to linear feature selective models (such as those using regional volume in Raz et al., 2005, 2010), our DL-architectures are trained end-to-end, and thus can incorporate information from diverse neural features, including volume, but also regionspecific sizes and shapes, tissue structures etc. Within our model those features can be nonlinearly related and weighted, and lastly, our multi-modal MLens leverage this capacity by incorporating complementary image-contrasts.

Similarly, in contrastive relevance maps, we found that heightened DBA values for subjects with type 2 diabetes displayed regions that corresponds to findings of recent animal models (Muramatsu et al., 2018) and known diabetes-associated degenerations in the sensorimotor areas in humans (Ferris et al., 2020). Moreover, our results support previous findings in 
diffusion imaging studies of changes in fiber bundles of the cingulum (Cui et al., 2020; Hoogenboom et al., 2014) and neighboring corpus callosum (Yu et al., 2019). That these findings appeared only significant in T1-weighted images, and not, as expected in FLAIR, might be due to the small sample size in the hold-out subset in combination with the less specific contrast of FLAIR in the absence of lesions. However, we found a strong spatial correspondence between the t-maps of both modalities.

We conclude that normal and pathologically driven aging is not exclusively represented in selective features (e.g., in the decline of regional volume) but also in diverse neurostructural properties accentuated by different MRI sequences, throughout the whole brain. More specifically, our analysis pipeline revealed that an individual's structural MRI carries not only global, macrostructural hints towards its age trajectory, but also reliable information on agerelated, subtle grey and white matter changes that occur all over the brain. While the limited image resolution does not offer explanations at the cellular level, those ubiquitous, rather subtle changes stem most likely from micro-changes, including oxidative stress, DNA damage, cell death and inflammation, in neuronal, vascular and glial compartments of the brain (Cole \& Franke, 2017; Pluvinage \& Wyss-Coray, 2020) that eventually alter the magnetic properties and thus image contrasts of the respective sequences. We can further infer that all brain regions and different neural properties that are highlighted with the different MRI sequences are predictive w.r.t. age, i.e., the aging process emerges in all these modalities. This calls for a multi-modal approach towards brain-aging rather than restricting this foundational phenomenon to selective neural variables such as grey matter volume, and acknowledges the capability of common structural MRI to reveal not only gross anatomical changes but also subtle microstructural changes with advancing age.

\section{The benefit of multi-level ensemble models}

Both types of MLens performed close to the state-of-the-art in the domain of BA prediction. Note that small performance differences might stem from our smaller dataset with a large agerange in comparison to studies that used, e.g., ukbiobank data ( $\mathrm{n}>14,000 \mathrm{MRIs}$, age range 
44-81 years; e.g., Peng et al., 2021, MAE $=2.14$ ). With our MLens we demonstrated that i) ensembles are performing better than their base models, and ii) MLens integrating diverse input features, here MRI sequences and brain regions, perform even better than ensembles that are only trained on one of these features.

On a methodological side, this shows that due to the feature selective training the model is prone to specialize on properties inherent to the respective feature (e.g., a brain region). Splitting the brain in sub-regions and feeding them to different models seems to push the respective models (here MLens type ii) to specialize on the characteristics of each brain region rather than learning filters that are generally usable across the whole brain, however, this needs to be tested systematically.

The variability of predictions between different $\mathrm{DL}$ models (here defined as the uncertainty between base models) with an identical architecture and training on the same data, underlines the importance of the aggregation over a set of models (i.e., an ensemble) to reduce both the variance and biases of single networks. In summary, MLens can not only compensate for the stochasticity of single DL models, but also provide estimates of model certainty and insights on the relationship of input features and prediction.

\section{Brain-age predictions and their association with other}

\section{biomarkers}

To investigate biological determinants of BA, we showed in an additional exploratory analysis that DBA was associated with cardiovascular risk factors such as BMI, waist-to-hip-ratio and type 2 diabetes. Notably, we found that many of these associations depend on the age of participants. For instance, despite the smaller sample size in our younger (healthy) cohort, we discovered a high correlation between $\mathrm{BMI}$ and the estimated $\mathrm{BA}$ (age $<40$ years), which was also reported in Kolenic et al. (2018) for younger participants with first-episode psychosis (1835 years). Also in mid-aged participants (40-60 years) we saw a significant correlation, for 
whom previous studies found higher BMI to be associated with cortical thinning (Shaw et al., 2018). Similar to previous findings (Kharabian Masouleh et al., 2016), also in the older cohort (60-80 years), a positive relationship appeared. Overall, with age the association between BA and BMI becomes weaker. Also, we found the positive correlation between DBA and type 2 diabetes, which was reported in Franke et al. (2013), and the corresponding relevance map analysis showed overlapping evidence w.r.t. GM changes as discussed above. Blood glucose levels (here HbA1c) showed relative consistent association across the cohort. With the estimates of MLens type $\mathrm{i}$ this association could also be seen in the $20-35$ years old, a result that corresponds to recent findings showing a negative relationship $\mathrm{HbA} 1 \mathrm{c}$ and $\mathrm{WM}$ integrity in young, non-diabetic (i.e., healthy) adults (mean age 28.8 years, $\mathrm{HbA} 1 \mathrm{c}<5.7 \%$; Repple et al., 2021), motivating further investigations. Overall, we found similar relationships of DBA and various clinical markers as summarized in Franke \& Gaser (2019), but not regarding ApoE-4 (cf. Raz et al., 2010). The found association between DBA and gender should be taken with caution, since demographic factors might have influenced the cohort composition in different age groups. Also, the gender difference is typically most pronounced in younger ages (Gur et al., 2002), while with menopause it appears to become smaller, brain-region specific (e.g., (Raz et al., 2010; Ritchie et al., 2018)) or is even absent (Jäncke et al., 2015). While we found a consistent, slightly negative trend (age $>25$ years) between DBA and cognitive performance, the correlation was not significant for most age strata; however, this association has been reported to be more pronounced in patients with $A D$ or mild cognitive impairments (Gaser et al., 2013; Liem et al., 2017). Note that we excluded participants with AD and other neurodegenerative diseases from this study, in which the relationship of DBA to cognitive performance, but also to associated biomarkers such as ApoE14 (see above) might be more pronounced. A very robust positive correlation, nearly across the full age range was found between the WM lesion-load and DBA. The typical accumulation of WM lesions with higher age as well as their pathological consequences are widely known (Beck et al., 2021; Dinsdale et al., 2021), and consequently and conversely, validates the BA models, while in parallel, this 
highlights the possibility that typical and pathological aging share similar fundamental mechanisms.

Clearly, these results indicate that $\mathrm{BA}$ is a reliable imaging marker reflecting biological plausible age-related neural changes. As deviations from the chronological age correlate with known risk factors for brain damage, BA can be considered as a biomarker of the brain health status of a person.

\section{Limitations and future research}

Several limitations need to be considered. First, despite the local information we receive with the LRP heatmaps, they do not explain per se what the biological mechanisms are that made the respective highlighted area relevant to the model. For instance, when considering relevant voxels around ventricles, we do not know whether a model tracks the size of a ventricle or potentially alterations at the tissue around it, or both. Further developments in interpretation algorithms, such as LRP could allow the detection of interactions between local and global relevance structures and in addition reveal causal relationships beyond correlation. Second, similar to (Levakov et al., 2020), we found that aggregating relevance maps compensated for the observed variability between heatmaps of single base models (for a discussion see Levakov et al., 2020). However, aggregation techniques can also cause information loss, for instance, not all of the base models within an ensemble might detect all WM lesions in an image. Third, the age distribution in the LIFE MRI dataset is non-uniform, with a majority of participants being 65 to 75 years of age. This introduces a bias in the training dataset. Although our ensemble architectures compensate for the prediction bias towards the distribution mean, this tendency could not be fully eliminated. The assessment of the covariate shift (e.g., Sugiyama et al., 2007), nonlinear head-models, and over- or undersampling techniques, combined with data augmentation could be further means to tackle this bias. Fourth, in future research one could run several cluster analyses to find common relevance patterns within, for instance, participants with certain pathologies or between different age 
groups. These could then be related to interpretable structural properties, such as cortical thickness (Frangou et al., 2021). Finally, the majority of studies cannot afford to scan thousands of participants. To make the presented explanation pipeline more sustainable, one could explore transfer learning techniques to adapt the pre-trained models to smaller datasets and different (target) variables. Since our approach makes it possible to combine information from different modalities and single out regions which show alterations in these modalities, one might also extend it to incorporating further imaging measures, e.g., diffusion imaging or resting-state studies in fMRI or EEG.

\section{Conclusion}

While certain brain areas shrink in volume more dramatically with older age than others, aging processes emerge in the whole brain. Their progress and pace can now be accurately captured and interpreted by $\mathrm{DL}$ ensembles from various brain regions and structural MRI modalities (T1, FLAIR, SWI), proposing that higher age and the presence of cardiovascular risk factors contributes to regionally pronounced yet ubiquitous changes in the brain. Employing the LRP interpretation algorithm, estimates of brain-aging can thus be related back to established, gross but also subtle, most likely microstructural biological markers of the aging process. This bias-free computational approach yields insights into the global nature of brain aging as well as pathomechanisms. Finally, due to its generalizability, this approach can be broadly applied across clinical neuroscience, galvanizing the generation of data-driven hypotheses and boosting its applications in personalized medicine (Binder et al., 2021; Esteva et al., 2021; Stenzinger et al., 2021). 


\section{Materials and methods}

\section{Data acquisition}

The LIFE Adult study (Loeffler et al., 2015), a population-based cohort study, encompasses dense clinical screenings of more than 10,000 participants coming from the area of Leipzig, Germany. Among others, the screening included measures of height, weight, blood pressure, blood-based biomarkers, cognitive performance and questionnaire batteries on mental health, and lifestyle (for more details see: Loeffler et al., 2015).

\section{Study sample and exclusion criteria}

Of the more than 10,000 subjects of the LIFE Adult study, 2637 participants underwent a 1hour MRI recording session at baseline. Of those participants with MR-scans, 621 participants were excluded mainly due to pathologies, leaving 2016 subjects for further analysis (age range 18-82 years, mean $n_{\text {age }}=57.32$, median $n_{\text {age }}=63.0 ; \mathrm{n}_{\text {female }}=946$; see Fig. $\mathbf{S 1}$ in supplementary material). Partially overlapping exclusion criteria were previous strokes $(n=54)$, excessive brain lesions rated by trained medical staff $(n=114)$, including white matter (WM) lesions rated with a Fazekas (Fazekas et al., 1987) score of $3(n=44)$, radiological diagnosis of brain tumor $(n=22)$, diagnosis of multiple sclerosis $(n=5)$, epilepsy $(n=27)$, cancer treatment in the last 12 months ( $n=109)$, centrally active medication $(n=275)$, cognitive impairments indicated by a MMSE score $<26(n=80)$, and poor quality MRIs (failing a visually quality check, e.g., regarding motion artefacts, $n=41)$.

\section{MRI data}

MRI data was acquired in a 1-hour recording session using a 32-channel head coil in a 3T Siemens Verio scanner. Various MRI sequences were applied (see Loeffler et al., 2015). For this study, we trained models on three MRI sequences used in clinical settings: i) structural 
T1-weighted images were taken with an MP-RAGE sequence $(1 \mathrm{~mm}$ isotropic voxels, 176 slices, $\mathrm{TR}=2300 \mathrm{~ms}, \mathrm{TE}=2.98 \mathrm{~ms}, \mathrm{TI}=900 \mathrm{~ms}$ ) which serves to quantify cerebrospinal fluid, white and gray matter. ii) Fluid-attenuated inversion recovery images (FLAIR) were acquired ( $1 \mathrm{~mm}$ isotropic voxels, 192 slices, TR=5000 ms, TE=395 ms, TI=1800 ms). FLAIR is highly sensitive towards lesions in the WM, which are known to accumulate with age (Beck et al., 2021; Ge et al., 2002; Tang et al., 1997). Lastly, iii) susceptibility-weighted magnitude images (SWI) are used to detect iron-deposits in the basal-ganglia (Bekiesinska-Figatowska et al., 2013; Pfefferbaum et al., 2009), which could be linked to neurodegeneration and cognitive decline (Du et al., 2018; Haller et al., 2010; G. E. C. Thomas et al., 2020), and are used to discover brain hemorrhages. SWIs were recorded with a T2*-weighted pulse sequence $(0.8 \mathrm{x}$ $0.7 \times 2.0 \mathrm{~mm}$ non-isotropic voxels, 64 slices, TR=28 ms, TE=20 ms).

\section{MRI preprocessing}

MRIs of the three sequences (T1, FLAIR, SWI) were saved in three processing stages: raw, freesurfer volume (recon-all, FreeSurfer 5.3.0; Fischl, 2012), and MNI stage (MNI152; Fonov et al., 2011), 2mm; via ANTs 2.2, (Tustison et al., 2020). In the freesurfer volume stage, FLAIR and SWI images were linear registered (linear interpolation; ANTs 2.2) to the corresponding space of the T1-weighted images ('brain.finalsurf.mgz'). For more details see the supplementary methods. For memory and processing efficiency, all images in all stages were pruned, i.e., their background was maximally removed, while keeping the same volume shape in the respective stage and, for raw images, respective sequence across all participants. These minimally-sized volumes were constrained to have a 2-voxel margin around the full brain of the largest brain in the whole dataset in the respective stage and sequence. Moreover, the image data was compressed by clipping upper intensity values to $383(255+50 \%)$, which affected an insignificant number of voxels $(<0.001 \%)$, and subsequently, by re-normalizing the data between 0-255 (i.e., into $2^{8}$ discrete intensity values per voxel). The re-normalized 
images were then processed as memory efficient arrays of single-byte, unsigned integers (here: uint8 type numpy 1.18.1 arrays; Harris et al., 2020).

\section{Prediction model (MRI data)}

\section{Model architecture}

Ensembles have been shown to predict more accurately and reduce model biases (Dietterich, 2000), also in the domain of BA prediction (Couvy-Duchesne et al., 2020; Dinsdale et al., 2021; Jonsson et al., 2019; Levakov et al., 2020; Peng et al., 2021). The individual predictions of the base models were used to train and evaluate a linear head model of the respective subensemble, leading to a weighted prediction of the whole ensemble. Subsequently, an additional linear top-head model was trained to aggregate predictions over those subensembles (see the following paragraphs, and Fig. 1).

\section{Base model}

The base model architecture was a 3D convolutional neural network (3D-CNN; Cole et al., 2017; Ji et al., 2013; LeCun et al., 1989; Lecun et al., 1998), implemented in native Keras 2.3.1 (Chollet, 2015). Base models were tested with two intermediate activation functions: i) the commonly applied rectified linear units (ReLUs), and ii) leaky ReLUs, which promise to overcome some of the drawbacks of absent gradients in standard ReLUs resulting from the background of MRIs, i.e., zero value input during training (Maas et al., 2013). From bottom up, the network consists of 5 convolutional blocks (ConvB), each starting with a convolutional layer $\left(\mathrm{n}_{\text {filters }} \in[16,16,32,64,32]\right.$, size $\left.\mathrm{kernel} \in\left[3^{3}, 3^{3}, 3^{3}, 3^{3}, 1^{3}\right]\right)$, followed by leaky ReLUs $(\forall$ leakyReLU, alpha $=0.2)$, and a 3D-max pool pool layer $\left(\forall \operatorname{maxpooL}\right.$, size $_{\text {pool }}=3^{3}$, stride $=$ $2^{3}$ ). Then the signal was flattened to a $1-D$ vector, and during training a dropout layer (rate $=$ 0.5) was applied. Finally, a fully connected layer (size $=64$ ) with (leaky) ReLUs propagated the signal to the linear output neuron. The bias at the linear output layer was set to the target 
mean $\bar{y}$ of the dataset (i.e., mean $_{\mathrm{age}}=57.32$ ), all other biases were randomly initialized around zero (Keras' default). The network was trained to minimize the mean squared error (MSE)

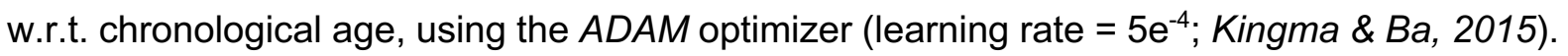
The data for the base models were split to a training, validation and test set ( $8: 1: 1$ ratio). The training process on the training set was monitored on the validation set. The reported model performances are the results of its evaluation on the test set, and are given as the mean absolute error (MAE) for better interpretability.

\section{Model ensembles}

Two types of MLens were trained: The first type consists of 3 sub-ensembles for $3 \mathrm{MRI}$ sequences (T1, FLAIR, SWI), respectively. Each sub-ensemble has 10 base models that were independently trained on the same training data (whole brain data in freesurfer volume stage of its respective MRI sequence). Then, a linear head model with weight regularization, i.e. ridge regression (alpha $=1$.) implemented in scikit-learn 0.22.1 (Buitinck et al., 2013), was trained on the predictions of the 10 base models per sub-ensemble on the validation set (shape of $X_{\text {train, head-model }}: N_{\text {val }} \times 10$ ), and evaluated on the test set (shape of $X_{\text {test, head-model }}: N_{\text {test }}$ $x$ 10). The resulting predictions of these 3 sub-ensembles on the test set were then used to train yet another head model in a 5-fold cross-validation approach to obtain aggregated predictions across all MRI sequences.

For the second MLens type, the MRI data (in MNI stage) was additionally masked in three different brain regions defined by the three complementary atlases (see Supplementary Material: Brain atlases). For each combination of region and MRI sequence $(3 \times 3), 5$ base models were trained, leading to a total of 45 base models. For each such combinatorial pair, its base model predictions were first aggregated with a linear head model (as above). Then, a linear top-head model combined these sub-ensemble predictions on the test set in the above mentioned 5-fold-cross-validation fashion to receive predictions across all input feature pairs (Fig. 1). 
Both MLens types (i, ii) can be conceptualized as neural additive models (Agarwal et al., 2020; Hastie \& Tibshirani, 1990), i.e. sub-parts of the ensemble are trained on different input features.

To receive an age estimate for each subject, the training procedure was run 10 times, such that each subject lies once in the test set. In each of the runs the MLens models were reinitiated.

\section{Estimation of model uncertainty}

Model certainty was measured subject-wise on both model levels, over each sub-ensemble and across them. That is, on the sub-ensemble level, model (un-)certainty is expressed in the width of the $95 \%$ - confidence interval around the mean prediction of all its base models for each subject. Similarly, the width of the $95 \%$ - confidence interval across the predictions of all sub-ensembles indicates the overall (un-)certainty of the MLens. Note, the latter could also be interpreted as information gain across input features.

\section{Prediction analyzer: Layer-wise Relevance Propagation}

\section{Simulation study on LRP for regression}

We created two-dimensional images of tori on black backgrounds at an intensity range comparable to T1-weighted MRIs that exhibited inner and outer surface atrophies as a linear function of their age (20-80 years) with a normally distributed variance, to simulate cortical atrophy and enlargement of cerebrospinal fluid space. Additionally, we simulated that the older a torus was, the more lesions it accumulated within its body, appearing as image hyperintensities. In contrast to the atrophies, this accumulation of lesions was non-linearly increasing with age (i.e., onset of linear increase at age 40), also with a normally distributed variance. For each torus, the location of atrophies and lesions were known allowing for the evaluation of the sensitivity of the model represented in the relevance maps. For the image 
details, please see the openly available code (https://github.com/SHEscher/XDLreg). We created 2000 tori, with a similar age-distribution as in the LIFE MRI sample. On this dataset, we then trained a 2D-version of the CNN as described above. Finally, LRP heatmaps were created on samples of the corresponding test set similar as described in following section. Since these heatmaps served only a qualitive analysis, we did not run statistical tests between them as we did for the MRI case.

\section{LRP for the MRI-based multi-level ensembles}

LRP was applied on the trained base models in one ensemble of each type (via iNNvestigate1.08; Alber et al., 2019), using the best-practice, composite rules (Kohlbrenner et al., 2020; Montavon et al., 2019) of LRP for CNNs (alpha = 1) implemented in iNNvestigate as "LRPSequentialPresetA". Note that we ran the LRP analysis only on models with ReLU activation functions, as it is recommended in iNNvestigate. LRP, which is usually employed in classification tasks, can be simply adapted to a regression problem by applying its algorithm starting at the single output neuron of the regression model. This is analogous to choosing the output neuron representing the ground-truth label of a given sample in a classification task. Differences are the task-specific objective function and the bias at the output layer, which we set to the distribution mean.

For the evaluation of the heatmaps, we took the average of the various relevance maps across base models similar to (Levakov et al., 2020). For between-subject analyses, we warped the subject respective heatmaps to MNI space. Relevance map aggregations within each subject were performed subsequently. The contribution of individual brain-regions to the model prediction was evaluated by mapping the LRP heatmaps to the merged brain-atlas, and the Juelich histological atlas (see Supplementary Material: Brain atlases). Additionally, we ran significance tests on the relevance maps with FSL 5.0 .8 (randomise function; using 5000 permutations and threshold-free cluster enhancement, TFCE) to determine brain areas which were statistically relevant for the BA prediction (Jenkinson et al., 2012). This was done, across 
all participants on their absolute aggregated relevance maps (one-sample t-test). Contrastive relevance maps (unpaired two-sample t-test) were computed in a young (age $\leq 40$ years) versus elderly (age $\geq 60$ years) group on their signed aggregated relevance maps. In older adults (age $\geq 50$ years), we analyzed in which brain regions relevance is attributed as function of the diverging (or delta) BA (DBA := ypredicted-age - ytrue-age) independent of chronological age. That is, we ran a generalized linear model (GLM; FSL 5.0.8, randomize), with relevance maps as regressand, and DBA as regressor, while controlling for age as covariate. Additionally, we explored the role of a pathobiological biomarker (see the following section for more details), specifically type 2 diabetes mellitus on the BA estimates within a wider, older age range (5075 years), contrasting diabetics to healthy controls (unpaired two-sample t-test on their signed aggregated relevance maps). To increase the sample size for all tests, we combined relevance maps from the validation and test set.

\section{Brain-age as a biomarker}

As an exploratory analysis, we correlated (Pearson's R; scipy 1.4.1; Virtanen et al., 2020; Bonferroni-corrected) DBA with a set of variables known to relate to accelerated brain aging.

These included cardiometabolic risk factors (BMI, waist-to-hip-ratio, hyperlipidemia, hypertension, systolic blood pressure, type 2 diabetes, glycated hemoglobin), genetic factors (apolipoprotein epsilon 4 risk-allele, APoE4, which has been associated with AD; Strittmatter et al., 1993), gender, time of education, cognitive functioning (composite score of executive functions, memory and processing speed, as reported in Kharabian Masouleh et al. (2016) and Zhang et al. (2018), and neural integrity (here measured as the logarithm of the ratio between number of lesions and white matter volume). For this, we applied an overlapping sliding window approach over the full age range (width 10 years) to model age-related associations between DBA and the above-mentioned variables, and to minimize the effect of age on the prediction error itself. 


\section{Acknowledgements}

This work is supported by the European Union, European Regional Development Fund as part of the LIFE-LIFT project, and the Free State of Saxony within the framework of the excellence initiative, and LIFE-Leipzig Research Center for Civilization Diseases, University of Leipzig (project numbers 713-241202, 14505/2470), and by the German Research Foundation (project numbers 209933838 CRC1052 Obesity mechanisms A1 and WI 3342/3-1). Further support was provided by the German Ministry for Education and Research (BMBF) through Berlin Institute for the Foundations of Learning and Data (BIFOLD; refs. 01IS18025A and 01IS18037A), MALT III (ref. 01IS17058), Patho234 (ref. 031L0207D) and Transparent Medical Expert Companion (TraMeExCo, ref. 01IS18056A), European Union's Horizon 2020 research and innovation programme through Intelligent Total Body Scanner for Early Detection of Melanoma (iToBoS, grant agreement No 965221), as well as the Grants 01GQ1115 and 01GQ0850; and by Deutsche Forschungsgemeinschaft (DFG) under Grant Math+, EXC 2046/1, Project ID 390685689; by the Institute of Information \& Communications Technology Planning \& Evaluation (IITP) grant funded by the Korea Government (No. 2019-0-00079, Artificial Intelligence Graduate School Program, Korea University).

\section{Author Contributions}

S.M.H., K.R.M., A.V., W.S., and A.V.W. designed and discussed the project.

Responsible for data acquisition M.L., A.V., and A.V.W.

Data analysis was done by S.M.H. and F.B.

Writing and editing were done by S.M.H., F.B., S.L., K.R.M., A.V., and A.V.W.

S.M.H. made the figures and tables.

\section{Competing Interests Statement}

The authors declare no competing interests. 


\section{References}

Agarwal, R., Frosst, N., Zhang, X., Caruana, R., \& Hinton, G. E. (2020). Neural Additive Models: Interpretable Machine Learning with Neural Nets. ArXiv:2004.13912 [Cs, Stat]. http://arxiv.org/abs/2004.13912

Alber, M., Lapuschkin, S., Seegerer, P., Hägele, M., Schütt, K. T., Montavon, G., Samek, W., Müller, K.-R., Dähne, S., \& Kindermans, P.-J. (2019). INNvestigate Neural Networks! Journal of Machine Learning Research, 20(93), 1-8.

Bach, S., Binder, A., Montavon, G., Klauschen, F., Müller, K.-R., \& Samek, W. (2015). On Pixel-Wise Explanations for Non-Linear Classifier Decisions by Layer-Wise Relevance Propagation. PLOS ONE, 10(7), e0130140. https://doi.org/10.1371/journal.pone.0130140

Baehrens, D., Schroeter, T., Harmeling, S., Kawanabe, M., Hansen, K., \& Müller, K.-R. (2010). How to Explain Individual Classification Decisions. The Journal of Machine Learning Research, 11, 1803-1831.

Bashyam, V. M., Erus, G., Doshi, J., Habes, M., Nasralah, I., Truelove-Hill, M., Srinivasan, D., Mamourian, L., Pomponio, R., Fan, Y., Launer, L. J., Masters, C. L., Maruff, P., Zhuo, C., Völzke, H., Johnson, S. C., Fripp, J., Koutsouleris, N., Satterthwaite, T. D., ... Davatzikos, C. (2020). MRI signatures of brain age and disease over the lifespan based on a deep brain network and 14468 individuals worldwide. Brain, awaa160. https://doi.org/10.1093/brain/awaa160

Beck, D., de Lange, A.-M. G., Maximov, I. I., Richard, G., Andreassen, O. A., Nordvik, J. E., \& Westlye, L. T. (2021). White matter microstructure across the adult lifespan: A mixed longitudinal and cross-sectional study using advanced diffusion models and brain-age prediction. Neurolmage, 224, 117441. https://doi.org/10.1016/j.neuroimage.2020.117441

Bekiesinska-Figatowska, M., Mierzewska, H., \& Jurkiewicz, E. (2013). Basal ganglia lesions in children and adults. European Journal of Radiology, 82(5), 837-849. https://doi.org/10.1016/j.ejrad.2012.12.006

Binder, A., Bockmayr, M., Hägele, M., Wienert, S., Heim, D., Hellweg, K., Ishii, M., Stenzinger, A., Hocke, A., Denkert, C., Müller, K.-R., \& Klauschen, F. (2021). Morphological and molecular breast cancer profiling through explainable machine learning. Nature Machine Intelligence. https://doi.org/10.1038/s42256-021-00303-4

Böhle, M., Eitel, F., Weygandt, M., \& Ritter, K. (2019). Layer-Wise Relevance Propagation for Explaining Deep Neural Network Decisions in MRI-Based Alzheimerltextquotesingles Disease Classification. Frontiers in Aging Neuroscience, 11. https://doi.org/10.3389/fnagi.2019.00194

Buitinck, L., Louppe, G., Blondel, M., Pedregosa, F., Mueller, A., Grisel, O., Niculae, V., Prettenhofer, P., Gramfort, A., Grobler, J., Layton, R., Vanderplas, J., Joly, A., Holt, B., \& Varoquaux, G. (2013). API design for machine learning software: Experiences from the scikit-learn project. ArXiv:1309.0238 [Cs]. http://arxiv.org/abs/1309.0238

Chollet, F. (2015). Keras. https://keras.io/

Cole, J. H., \& Franke, K. (2017). Predicting Age Using Neuroimaging: Innovative Brain Ageing Biomarkers. Trends in Neurosciences, 40(12), 681-690. https://doi.org/10.1016/j.tins.2017.10.001

Cole, J. H., Poudel, R. P. K., Tsagkrasoulis, D., Caan, M. W. A., Steves, C., Spector, T. D., \& Montana, G. (2017). Predicting brain age with deep learning from raw imaging data results in a reliable and heritable biomarker. Neurolmage, 163, 115-124. https://doi.org/10.1016/j.neuroimage.2017.07.059

Couvy-Duchesne, B., Faouzi, J., Martin, B., Thibeau-Sutre, E., Wild, A., Ansart, M., Durrleman, S., Dormont, D., Burgos, N., \& Colliot, O. (2020). Ensemble Learning of Convolutional Neural Network, Support Vector Machine, and Best Linear Unbiased Predictor for Brain Age Prediction: ARAMIS Contribution to the Predictive Analytics Competition 2019 Challenge. Frontiers in Psychiatry, 11, 593336. 
https://doi.org/10.3389/fpsyt.2020.593336

Cui, Y., Tang, T.-Y., Lu, C.-Q., Cai, Y., Lu, T., Wang, Y.-C., Teng, G.-J., \& Ju, S. (2020). Abnormal Cingulum Bundle Induced by Type 2 Diabetes Mellitus: A Diffusion Tensor Tractography Study. Frontiers in Aging Neuroscience, 12, 594198. https://doi.org/10.3389/fnagi.2020.594198

Dadi, K., Varoquaux, G., Houenou, J., Bzdok, D., Thirion, B., \& Engemann, D. (2020). Beyond brain age: Empirically-derived proxy measures of mental health [Preprint]. https://doi.org/10.1101/2020.08.25.266536

Dietterich, T. G. (2000). Ensemble Methods in Machine Learning. Multiple Classifier Systems, 1-15.

Dinsdale, N. K., Bluemke, E., Smith, S. M., Arya, Z., Vidaurre, D., Jenkinson, M., \& Namburete, A. I. L. (2021). Learning patterns of the ageing brain in MRI using deep convolutional networks. Neurolmage, 224, 117401. https://doi.org/10.1016/j.neuroimage.2020.117401

Doraiswamy, M., Figiel, G. S., Husain, M. M., McDonald, W. M., Shah, S. A., Boyko, O. B., Ellinwood, E. H., Jr., \& Krishnan, K. R. R. (1991). Aging of the human corpus callosum: Magnetic resonance imaging in normal volunteers. The Journal of Neuropsychiatry and Clinical Neurosciences, 3(4), 392-397. https://doi.org/10.1176/jnp.3.4.392

Du, L., Zhao, Z., Cui, A., Zhu, Y., Zhang, L., Liu, J., Shi, S., Fu, C., Han, X., Gao, W., Song, T., Xie, L., Wang, L., Sun, S., Guo, R., \& Ma, G. (2018). Increased Iron Deposition on Brain Quantitative Susceptibility Mapping Correlates with Decreased Cognitive Function in Alzheimer's Disease. ACS Chemical Neuroscience, 9(7), 1849-1857. https://doi.org/10.1021/acschemneuro.8b00194

Earnest, M. P., Heaton, R. K., Wilkinson, W. E., \& Manke, W. F. (1979). Cortical atrophy, ventricular enlargement and intellectual impairment in the aged. Neurology, 29(8), 1138-1138. https://doi.org/10.1212/wnl.29.8.1138

Eitel, F., Soehler, E., Bellmann-Strobl, J., Brandt, A. U., Ruprecht, K., Giess, R. M., Kuchling, J., Asseyer, S., Weygandt, M., Haynes, J.-D., Scheel, M., Paul, F., \& Ritter, K. (2019). Uncovering convolutional neural network decisions for diagnosing multiple sclerosis on conventional MRI using layer-wise relevance propagation. Neurolmage: Clinical, 24, 102003. https://doi.org/10.1016/j.nicl.2019.102003

Esteva, A., Chou, K., Yeung, S., Naik, N., Madani, A., Mottaghi, A., Liu, Y., Topol, E., Dean, J., \& Socher, R. (2021). Deep learning-enabled medical computer vision. Npj Digital Medicine, 4(1), 5. https://doi.org/10.1038/s41746-020-00376-2

Fazekas, F., Chawluk, J., Alavi, A., Hurtig, H., \& Zimmerman, R. (1987). MR signal abnormalities at $1.5 \mathrm{~T}$ in Alzheimer's dementia and normal aging. American Journal of Roentgenology, 149(2), 351-356. https://doi.org/10.2214/ajr.149.2.351

Feng, X., Lipton, Z. C., Yang, J., Small, S. A., \& Provenzano, F. A. (2020). Estimating brain age based on a uniform healthy population with deep learning and structural magnetic resonance imaging. Neurobiology of Aging, 91, 15-25. https://doi.org/10.1016/j.neurobiolaging.2020.02.009

Ferris, J. K., Inglis, J. T., Madden, K. M., \& Boyd, L. A. (2020). Brain and Body: A Review of Central Nervous System Contributions to Movement Impairments in Diabetes. Diabetes, 69(1), 3-11. https://doi.org/10.2337/db19-0321

Fischl, B. (2012). FreeSurfer. Neurolmage, 62(2), 774-781. https://doi.org/10.1016/j.neuroimage.2012.01.021

Fonov, V., Evans, A. C., Botteron, K., Almli, C. R., McKinstry, R. C., \& Collins, D. L. (2011). Unbiased average age-appropriate atlases for pediatric studies. Neurolmage, 54(1), 313-327. https://doi.org/10.1016/j.neuroimage.2010.07.033

Frangou, S., Modabbernia, A., Williams, S. C. R., Papachristou, E., Doucet, G. E., Agartz, I., Aghajani, M., Akudjedu, T. N., Albajes-Eizagirre, A., Alnæs, D., Alpert, K. I., Andersson, M., Andreasen, N. C., Andreassen, O. A., Asherson, P., Banaschewski, T., Bargallo, N., Baumeister, S., Baur-Streubel, R., ... Dima, D. (2021). Cortical thickness across the lifespan: Data from 17,075 healthy individuals aged 3-90 years. 
Human Brain Mapping, hbm.25364. https://doi.org/10.1002/hbm.25364

Franke, K., \& Gaser, C. (2019). Ten Years of BrainAGE as a Neuroimaging Biomarker of Brain Aging: What Insights Have We Gained? Frontiers in Neurology, 10. https://doi.org/10.3389/fneur.2019.00789

Franke, K., Gaser, C., Manor, B., \& Novak, V. (2013). Advanced BrainAGE in older adults with type 2 diabetes mellitus. Frontiers in Aging Neuroscience, 5. https://doi.org/10.3389/fnagi.2013.00090

Gaser, C., Franke, K., Klöppel, S., Koutsouleris, N., \& and, H. S. (2013). BrainAGE in Mild Cognitive Impaired Patients: Predicting the Conversion to Alzheimer's Disease. PLoS ONE, 8(6), e67346. https://doi.org/10.1371/journal.pone.0067346

Ge, Y., Grossman, R. I., Babb, J. S., Rabin, M. L., Mannon, L. J., \& Kolson, D. L. (2002). Age-Related Total Gray Matter and White Matter Changes in Normal Adult Brain. Part I: Volumetric MR Imaging Analysis. American Journal of Neuroradiology, 23(8), 1327-1333.

Gur, R. C., Gunning-Dixon, F. M., Turetsky, B. I., Bilker, W. B., \& Gur, R. E. (2002). Brain Region and Sex Differences in Age Association With Brain Volume: A Quantitative MRI Study of Healthy Young Adults. The American Journal of Geriatric Psychiatry, 10(1), 72-80. https://doi.org/10.1097/00019442-200201000-00009

Haller, S., Bartsch, A., Nguyen, D., Rodriguez, C., Emch, J., Gold, G., Lovblad, K. O., \& Giannakopoulos, P. (2010). Cerebral Microhemorrhage and Iron Deposition in Mild Cognitive Impairment: Susceptibility-weighted MR Imaging Assessment. Radiology, 257(3), 764-773. https://doi.org/10.1148/radiol.10100612

Harris, C. R., Millman, K. J., van der Walt, S. J., Gommers, R., Virtanen, P., Cournapeau, D., Wieser, E., Taylor, J., Berg, S., Smith, N. J., Kern, R., Picus, M., Hoyer, S., van Kerkwijk, M. H., Brett, M., Haldane, A., del Río, J. F., Wiebe, M., Peterson, P., ... Oliphant, T. E. (2020). Array programming with NumPy. Nature, 585(7825), 357-362. https://doi.org/10.1038/s41586-020-2649-2

Hastie, T., \& Tibshirani, R. (1990). Generalized additive models. 10.

Hoogenboom, W. S., Marder, T. J., Flores, V. L., Huisman, S., Eaton, H. P., Schneiderman, J. S., Bolo, N. R., Simonson, D. C., Jacobson, A. M., Kubicki, M., Shenton, M. E., \& Musen, G. (2014). Cerebral White Matter Integrity and Resting-State Functional Connectivity in Middle-aged Patients With Type 2 Diabetes. Diabetes, 63(2), 728738. https://doi.org/10.2337/db13-1219

Jäncke, L., Mérillat, S., Liem, F., \& Hänggi, J. (2015). Brain size, sex, and the aging brain. Human Brain Mapping, 36(1), 150-169. https://doi.org/10.1002/hbm.22619

Jenkinson, M., Beckmann, C. F., Behrens, T. E. J., Woolrich, M. W., \& Smith, S. M. (2012). FSL. Neurolmage, 62(2), 782-790. https://doi.org/10.1016/j.neuroimage.2011.09.015

Ji, S., Xu, W., Yang, M., \& Yu, K. (2013). 3D Convolutional Neural Networks for Human Action Recognition. IEEE Transactions on Pattern Analysis and Machine Intelligence, 35(1), 221-231. https://doi.org/10.1109/TPAMI.2012.59

Jin, K., Zhang, T., Shaw, M., Sachdev, P., \& Cherbuin, N. (2018). Relationship Between Sulcal Characteristics and Brain Aging. Frontiers in Aging Neuroscience, 10, 339. https://doi.org/10.3389/fnagi.2018.00339

Jonsson, B. A., Bjornsdottir, G., Thorgeirsson, T. E., Ellingsen, L. M., Walters, G. B., Gudbjartsson, D. F., Stefansson, H., Stefansson, K., \& Ulfarsson, M. O. (2019). Brain age prediction using deep learning uncovers associated sequence variants. Nature Communications, 10(1). https://doi.org/10.1038/s41467-019-13163-9

Kharabian Masouleh, S., Arélin, K., Horstmann, A., Lampe, L., Kipping, J. A., Luck, T., Riedel-Heller, S. G., Schroeter, M. L., Stumvoll, M., Villringer, A., \& Witte, A. V. (2016). Higher body mass index in older adults is associated with lower gray matter volume: Implications for memory performance. Neurobiology of Aging, 40, 1-10. https://doi.org/10.1016/j.neurobiolaging.2015.12.020

Kingma, D. P., \& Ba, J. L. (2015). Adam: A Method for Stochastic Optimization. ICLR, 1-15. Kochunov, P., Mangin, J.-F., Coyle, T., Lancaster, J., Thompson, P., Rivière, D., Cointepas, Y., Régis, J., Schlosser, A., Royall, D. R., Zilles, K., Mazziotta, J., Toga, A., \& Fox, P. 
T. (2005). Age-related morphology trends of cortical sulci. Human Brain Mapping, 26(3), 210-220. https://doi.org/10.1002/hbm.20198

Kohlbrenner, M., Bauer, A., Nakajima, S., Binder, A., Samek, W., \& Lapuschkin, S. (2020).

Towards best practice in explaining neural network decisions with LRP. 2020 International Joint Conference on Neural Networks (IJCNN), 1-7.

Kolbeinsson, A., Filippi, S., Panagakis, Y., Matthews, P. M., Elliott, P., Dehghan, A., \& Tzoulaki, I. (2020). Accelerated MRI-predicted brain ageing and its associations with cardiometabolic and brain disorders. Scientific Reports, 10(1), 19940. https://doi.org/10.1038/s41598-020-76518-z

Kolenic, M., Franke, K., Hlinka, J., Matejka, M., Capkova, J., Pausova, Z., Uher, R., Alda, M., Spaniel, F., \& Hajek, T. (2018). Obesity, dyslipidemia and brain age in firstepisode psychosis. Journal of Psychiatric Research, 99, 151-158.

https://doi.org/10.1016/j.jpsychires.2018.02.012

Lapuschkin, S., Wäldchen, S., Binder, A., Montavon, G., Samek, W., \& Müller, K.-R. (2019). Unmasking Clever Hans predictors and assessing what machines really learn. Nature Communications, 10(1), 1096. https://doi.org/10.1038/s41467-019-08987-4

LeCun, Y., Boser, B., Denker, J. S., Henderson, D., Howard, R. E., Hubbard, W., \& Jackel, L. D. (1989). Backpropagation Applied to Handwritten Zip Code Recognition. Neural Computation, 1(4), 541-551. https://doi.org/10.1162/neco.1989.1.4.541

Lecun, Y., Bottou, L., Bengio, Y., \& Haffner, P. (1998). Gradient-based learning applied to document recognition. Proceedings of the IEEE, 86(11), 2278-2324. https://doi.org/10.1109/5.726791

LeMay, M. (1984). Radiologic changes of the aging brain and skull. American Journal of Roentgenology, 143(2), 383-389. https://doi.org/10.2214/ajr.143.2.383

Levakov, G., Rosenthal, G., Shelef, I., Raviv, T. R., \& Avidan, G. (2020). From a deep learning model back to the brain-Identifying regional predictors and their relation to aging. Human Brain Mapping, hbm.25011. https://doi.org/10.1002/hbm.25011

Liem, F., Varoquaux, G., Kynast, J., Beyer, F., Masouleh, S. K., Huntenburg, J. M., Lampe, L., Rahim, M., Abraham, A., Craddock, R. C., Riedel-Heller, S., Luck, T., Loeffler, M., Schroeter, M. L., Witte, A. V., Villringer, A., \& Margulies, D. S. (2017). Predicting brain-age from multimodal imaging data captures cognitive impairment. Neurolmage, 148, 179-188. https://doi.org/10.1016/j.neuroimage.2016.11.005

Loeffler, M., Engel, C., Ahnert, P., Alfermann, D., Arelin, K., Baber, R., Beutner, F., Binder, H., Brähler, E., Burkhardt, R., Ceglarek, U., Enzenbach, C., Fuchs, M., Glaesmer, H., Girlich, F., Hagendorff, A., Häntzsch, M., Hegerl, U., Henger, S., ... Thiery, J. (2015). The LIFE-Adult-Study: Objectives and design of a population-based cohort study with 10,000 deeply phenotyped adults in Germany. BMC Public Health, 15(1). https://doi.org/10.1186/s12889-015-1983-z

Maas, A. L., Hannun, A. Y., \& Ng, A. Y. (2013). Rectifier Nonlinearities Improve Neural Network Acoustic Models. In Proceedings of ICML 2013, 6.

Montavon, G., Binder, A., Lapuschkin, S., Samek, W., \& Müller, K.-R. (2019). Layer-Wise Relevance Propagation: An Overview. In Explainable Al: Interpreting, Explaining and Visualizing Deep Learning (pp. 193-209). Springer International Publishing. https://doi.org/10.1007/978-3-030-28954-6_10

Montavon, G., Samek, W., \& Müller, K.-R. (2018). Methods for interpreting and understanding deep neural networks. Digital Signal Processing, 73, 1-15. https://doi.org/10.1016/j.dsp.2017.10.011

Muramatsu, K., Ikutomo, M., Tamaki, T., Shimo, S., \& Niwa, M. (2018). Effect of streptozotocin-induced diabetes on motor representations in the motor cortex and corticospinal tract in rats. Brain Research, 1680, 115-126. https://doi.org/10.1016/j.brainres.2017.12.016

Peng, H., Gong, W., Beckmann, C. F., Vedaldi, A., \& Smith, S. M. (2021). Accurate brain age prediction with lightweight deep neural networks. Medical Image Analysis, 68, 101871. https://doi.org/10.1016/j.media.2020.101871

Pfefferbaum, A., Adalsteinsson, E., Rohlfing, T., \& Sullivan, E. V. (2009). MRI estimates of 
brain iron concentration in normal aging: Comparison of field-dependent (FDRI) and phase (SWI) methods. Neurolmage, 47(2), 493-500.

https://doi.org/10.1016/j.neuroimage.2009.05.006

Pluvinage, J. V., \& Wyss-Coray, T. (2020). Systemic factors as mediators of brain homeostasis, ageing and neurodegeneration. Nature Reviews Neuroscience, 21(2), 93-102. https://doi.org/10.1038/s41583-019-0255-9

Raz, N., Ghisletta, P., Rodrigue, K. M., Kennedy, K. M., \& Lindenberger, U. (2010).

Trajectories of brain aging in middle-aged and older adults: Regional and individual differences. 11.

Raz, N., Lindenberger, U., Rodrigue, K. M., Kennedy, K. M., Head, D., Williamson, A., Dahle, C., Gerstorf, D., \& Acker, J. D. (2005). Regional Brain Changes in Aging Healthy Adults: General Trends, Individual Differences and Modifiers. Cerebral Cortex, 15(11), 1676-1689. https://doi.org/10.1093/cercor/bhi044

Repple, J., Karliczek, G., Meinert, S., Förster, K., Grotegerd, D., Goltermann, J., Redlich, R., Arolt, V., Baune, B. T., Dannlowski, U., \& Opel, N. (2021). Variation of HbA1c affects cognition and white matter microstructure in healthy, young adults. Molecular Psychiatry, 26(4), 1399-1408. https://doi.org/10.1038/s41380-019-0504-3

Ritchie, S. J., Cox, S. R., Shen, X., Lombardo, M. V., Reus, L. M., Alloza, C., Harris, M. A., Alderson, H. L., Hunter, S., Neilson, E., Liewald, D. C. M., Auyeung, B., Whalley, H. C., Lawrie, S. M., Gale, C. R., Bastin, M. E., Mclntosh, A. M., \& Deary, I. J. (2018). Sex Differences in the Adult Human Brain: Evidence from 5216 UK Biobank Participants. Cerebral Cortex, 28(8), 2959-2975. https://doi.org/10.1093/cercor/bhy109

Samek, W., Montavon, G., Lapuschkin, S., Anders, C. J., \& Muller, K.-R. (2021). Explaining Deep Neural Networks and Beyond: A Review of Methods and Applications. Proceedings of the IEEE, 109(3), 247-278. https://doi.org/10.1109/JPROC.2021.3060483

Samek, W., Montavon, G., Vedaldi, A., Hansen, L. K., \& Müller, K.-R. (2019). Explainable Al: interpreting, explaining and visualizing deep learning (Vol. 11700). Springer Nature.

Shaw, M. E., Sachdev, P. S., Abhayaratna, W., Anstey, K. J., \& Cherbuin, N. (2018). Body mass index is associated with cortical thinning with different patterns in mid- and latelife. International Journal of Obesity, 42(3), 455-461. https://doi.org/10.1038/ijo.2017.254

Simonyan, K., Vedaldi, A., \& Zisserman, A. (2014). Deep inside convolutional networks: Visualising image classification models and saliency maps. ICLR, 1-8.

Smilkov, D., Thorat, N., Kim, B., Viégas, F., \& Wattenberg, M. (2017). SmoothGrad: Removing noise by adding noise. ArXiv:1706.03825 [Cs, Stat]. http://arxiv.org/abs/1706.03825

Stenzinger, A., Alber, M., Allgäuer, M., Jurmeister, P., Bockmayr, M., Budczies, J., Lennerz, J., Eschrich, J., Kazdal, D., Schirmacher, P., Wagner, A. H., Tacke, F., Capper, D., Müller, K.-R., \& Klauschen, F. (2021). Artificial intelligence and pathology: From principles to practice and future applications in histomorphology and molecular profiling. Seminars in Cancer Biology, S1044579X21000341. https://doi.org/10.1016/j.semcancer.2021.02.011

Strittmatter, W. J., Weisgraber, K. H., Huang, D. Y., Dong, L. M., Salvesen, G. S., PericakVance, M., Schmechel, D., Saunders, A. M., Goldgaber, D., \& Roses, A. D. (1993). Binding of human apolipoprotein E to synthetic amyloid beta peptide: Isoform-specific effects and implications for late-onset Alzheimer disease. Proceedings of the National Academy of Sciences, 90(17), 8098-8102. https://doi.org/10.1073/pnas.90.17.8098

Sudlow, C., Gallacher, J., Allen, N., Beral, V., Burton, P., Danesh, J., Downey, P., Elliott, P., Green, J., Landray, M., Liu, B., Matthews, P., Ong, G., Pell, J., Silman, A., Young, A., Sprosen, T., Peakman, T., \& Collins, R. (2015). UK Biobank: An Open Access Resource for Identifying the Causes of a Wide Range of Complex Diseases of Middle and Old Age. PLOS Medicine, 12(3), e1001779. 
https://doi.org/10.1371/journal.pmed.1001779

Sugiyama, M., Krauledat, M., \& Müller, K.-R. (2007). Covariate shift adaptation by importance weighted cross validation. Journal of Machine Learning Research, 8(5).

Sundararajan, M., Taly, A., \& Yan, Q. (2017). Axiomatic Attribution for Deep Networks. In D. Precup \& Y. W. Teh (Eds.), Proceedings of the 34th International Conference on Machine Learning, ICML 2017, Sydney, NSW, Australia, 6-11 August 2017 (Vol. 70, pp. 3319-3328). PMLR. http://proceedings.mlr.press/v70/sundararajan17a.html

Tang, Y., Nyengaard, J. R., Pakkenberg, B., \& Gundersen, H. J. G. (1997). Age-Induced White Matter Changes in the Human Brain: A Stereological Investigation. Neurobiology of Aging, 18(6), 609-615. https://doi.org/10.1016/S01974580(97)00155-3

Thomas, A. W., Heekeren, H. R., Müller, K.-R., \& Samek, W. (2019). Analyzing neuroimaging data through recurrent deep learning models. Frontiers in Neuroscience, 13, 1321.

Thomas, G. E. C., Leyland, L. A., Schrag, A.-E., Lees, A. J., Acosta-Cabronero, J., \& Weil, R. S. (2020). Brain iron deposition is linked with cognitive severity in Parkinson's disease. Journal of Neurology, Neurosurgery \& Psychiatry, 91(4), 418-425. https://doi.org/10.1136/jnnp-2019-322042

Tustison, N. J., Cook, P. A., Holbrook, A. J., Johnson, H. J., Muschelli, J., Devenyi, G. A., Duda, J. T., Das, S. R., Cullen, N. C., Gillen, D. L., Yassa, M. A., Stone, J. R., Gee, J. C., Avants, B. B., \& the Alzheimer's Disease Neuroimaging Initiative. (2020). ANTSX: A dynamic ecosystem for quantitative biological and medical imaging [Preprint]. Radiology and Imaging. https://doi.org/10.1101/2020.10.19.20215392

Virtanen, P., Gommers, R., Oliphant, T. E., Haberland, M., Reddy, T., Cournapeau, D., Burovski, E., Peterson, P., Weckesser, W., Bright, J., van der Walt, S. J., Brett, M., Wilson, J., Millman, K. J., Mayorov, N., Nelson, A. R. J., Jones, E., Kern, R., Larson, E., ... van Mulbregt, P. (2020). SciPy 1.0: Fundamental algorithms for scientific computing in Python. Nature Methods, 17(3), 261-272. https://doi.org/10.1038/s41592-019-0686-2

Yu, X., Jiaerken, Y., Xu, X., Jackson, A., Huang, P., Yang, L., Yuan, L., Lou, M., Jiang, Q., \& Zhang, M. (2019). Abnormal corpus callosum induced by diabetes impairs sensorimotor connectivity in patients after acute stroke. European Radiology, 29(1), 115-123. https://doi.org/10.1007/s00330-018-5576-y

Zeiler, M. D., \& Fergus, R. (2014). Visualizing and Understanding Convolutional Networks. Proc. of European Conference on Computer Vision (ECCV), 818-833.

Zhang, R., Beyer, F., Lampe, L., Luck, T., Riedel-Heller, S. G., Loeffler, M., Schroeter, M. L., Stumvoll, M., Villringer, A., \& Witte, A. V. (2018). White matter microstructural variability mediates the relation between obesity and cognition in healthy adults. Neurolmage, 172, 239-249. https://doi.org/10.1016/j.neuroimage.2018.01.028

Zintgraf, L. M., Cohen, T. S., Adel, T., \& Welling, M. (2017). Visualizing Deep Neural Network Decisions: Prediction Difference Analysis. International Conference on Learning Representations (ICLR), 2017. 